ISSN (print) o867-2008 / ISSN (online) 2391-75 I 2

DOI: http://dx.doi.org/10.12775/OM.2019.005

KARL BORCHARDT*

Monumenta Germaniae Historica, München

Ludwigstraße 16

D-80539 München

Germany

karl.borchardt@mgh.de

\title{
FRATRES CLERICI UND BENEFICIA ECCLESIASTICA IM MITTELALTERLICHEN JOHANNITERPRIORAT ALAMANIA
}

\section{KEYWORDS}

history; the Middle Ages; military orders; Priory of Alamania; commanderies; ecclesiastical benefices; ius patronatus; incorporation

\section{Abstract \\ Fratres Clerici and Beneficia Ecclesiastica in the Medieval Hospitaller Priory of Alamania}

The Hospitallers acquired possessions in Latin Europe during the twelfth and thirteenth centuries first through donations, later on also by purchase, in order to support their activities in the Levant. These acquisitions included parishes, hospitals and castles, although their maintenance necessitated expenditures that reduced the surpluses the Hospitallers could use for the Levant. At the same time parishes and other ecclesiastical benefices were restructured according to the concepts of ius patronatus and incorporation. As a consequence, episcopal supervision was strengthened even for those parishes and benefices which were owned by the Hospitallers and other religious orders. In general, fratres clerici were of lesser importance in the Order than fratres milites. Nevertheless, they were eligible to become commanders or to administer commanderies for absentee commanders, and they were indispensable both for services in the Order's parish churches and for prayers on behalf of the souls of the Order's members and benefactors. During the later Middle Ages their role was strengthened especially in Cologne and in the two new Hospitaller foundations at Straßburg in I37I and Biel in I454/1455. After the Reformation the Order's priests received an exclusive right roughly one third of the commanderies.

* Professor emeritus. 


\section{Historische GRUNDlagen}

Is geistlicher Ritterorden zählten die Johanniter Besitz und Betrieb von
Pfarreien im lateinischen Abendland an sich nicht zu ihren zentralen Auf-
gaben, ebensowenig wie Besitz und Betrieb von Spitälern oder Burgen. Schenkungen jedoch konnten sie schlecht ablehnen, insbesondere wenn sich dadurch Überschüsse zum Nutzen des Heiligen Landes erzielen ließen. Bei Pfarreien in Dörfern und vor allem in Städten, wo die Bevölkerung stark wuchs, war das zweifellos der Fall. Schon im I 2. und erst recht seit dem I 3. Jahrhundert erwarben die Johanniter deshalb anfangs durch Schenkung, später gezielt durch Kauf im

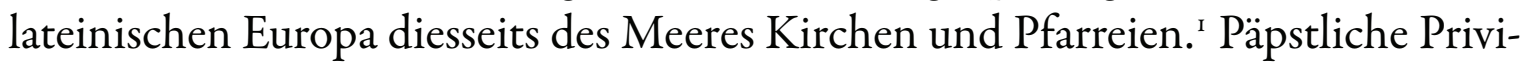
legien ermöglichten und ermutigten solche Erwerbungen, um den Orden für den Kampf gegen die Muslime jenseits des Meeres, in Outremer, zu stärken. Spätestens im I 3. Jahrhundert richtete der Johanniterorden bei einzelnen Häusern im Westen sogar Konvente ein, in denen mehrere Ordensbrüder - fratres, und zwar milites, clerici und servientes - auf Dauer lebten. ${ }^{2}$ Dort konnte man eintreten, gewöhnlich

I Grundlegend bleiben Walter G. Rödel, Das Großpriorat Deutschland des Johanniter-Ordens im Übergang vom Mittelalter zur Reformation anhand der Generalvisitationsberichte von 1494/95 und I540/4I (Köln: Wienand, I 972, 2. Aufl.); Johanna Maria van Winter, Sources Concerning the Hospitallers of St John in the Netherlands I4th-I8th Centuries, Studies in the History of Christian Thought 89 (Leiden-Boston-Köln: Brill, I 998); dazu Karl Borchardt, "Soll-Zahlen zum Personalstand der deutschen Johanniter vom Jahre I 367," Revue Mabillon 75, Nr. I 4 (2003): 83-I I 3 . Besonders gut ist inzwischen der Forschungsstand für die Ballei Sachsen, Mark Brandenburg, Wendland und Pommern: Brandenburgisches Klosterbuch: Handbuch der Klöster, Stifte und Kommenden bis zur Mitte des I6. Jahrbunderts, 2 Bde, hrsg. v. Heinz-Dieter Heimann, Klaus Neitmann, und Winfried Schich (Berlin: be.bra wissenschaft, 2007) für Werben, Tempelhof, Lietzen, Grüneberg, Soldin, Quartschen, Sonnenburg, Lagow; Mecklenburgisches Klosterbuch: Handbuch der Klöster, Stifte und Kommenden (Io.-I6. Jahrbundert), hrsg. v. Wolfgang Huschner, Ernst Münch, und Cornelia Neustadt (Rostock: Hinstorff, 2016) für Eichsen, Gardow, Kraak, Lychen, Mirow, Nemerow; Niedersächsisches Klosterbuch: Verzeichnis der Klöster, Stifte, Kommenden und Beginenhäuser in Niedersachsen und Bremen von den Anfängen bis I 810 , hrsg. v. Josef Dolle (Bielefeld: Verlag für Regionalgeschichte, 20 I 2) für Abbingwehr, Boekzetel, Bokelesch, Braunschweig, Bredehorn, Burlage, Burmönken, Dünebroek, Emmerstedt?, Esterwegen, Fallersleben, Gartow, Goslar, Hahn?, Hasselt, Heiselhusen, Hesel, Hoven, Inte, Jemgum, Klosterholte?, Lage, Langewische, Langholt, Lemwerder, Muhde, Oldenburg, Roddens, Strückhausen, Süpplingenburg, Tjüchen, Versfleth, Witleke und für Templer in Braunschweig, Emmerstedt, Hameln?, Hildesheim (fiktiv), Hoven, Süpplingenburg, Tempelhof.

2 Wichtige Übersichten bieten Helvetia Sacra, Abteilung 4, Bd. 7, Tl. I, Die Johanniter, die Templer, der Deutsche Orden. die Lazariter und Lazariterinnen, die Pauliner und die Serviten in der Schweiz, hrsg. v. Petra Zimmer und Patrick Braun (Basel: Schwabe, 2006) für das Großpriorat selbst sowie für die Kommenden Basel, Biberstein, Biel, Bubikon, Freiburg im Üchtland, Hohenrain, Klingnau, Küsnacht, Leuggern, Buchsee, Reiden, Rheinfelden, Thunstetten, Tobel und Wädenswil; Westfälisches Klosterbuch: Lexikon der vor I 8 Is errichteten Stifte und Klöster 
im Gegenzug für eine Schenkung. Die Ordensoberen konnten aber auch fratres versetzen, damit beliebte Konvente nicht überlastet wurden. Oder sie konnten Ordensbrüder zur Belohnung oder Bestrafung in bestimmte Konvente einweisen. Geleitet wurden die Ordensniederlassungen in weltlichen Angelegenheiten durch den Komtur (preceptor). Wenn es in einem Haus mehrere Ordensgeistliche gab, stand an ihrer Spitze oft ein Prior. Vorbild war hier das Haupthaus in der Levante, wo der Meister neben anderen Amtsträgern für die Aufsicht über die Ordensgeistlichen ebenfalls einen Prior unter sich hatte. Beide Prioren, der im Haupthaus und der im einzelnen Konvent, waren Geistliche und dürfen nicht verwechselt werden mit den rund 20 Prioren für einzelne Provinzen und Regionen im lateinischen Abendland, darunter eben dem Prior von Alamania; letztere gingen fast durchweg aus dem Kreise der im Orden tonangebenden Ritterbrüder hervor.

Ebenfalls im I 2. und I 3. Jahrhundert strukturierte das kanonische Recht das Institut der Pfarrei neu. Das war notwendig angesichts des Bevölkerungswachstums im lateinischen Abendland. Unvermeidlich wurden zahlreiche neue Pfarreien gegründet, nicht nur in Dörfern, sondern auch in den überall wie Pilze aus dem Boden schießenden Städten. Stadtherren betrauten gerne Klöster, Stifte oder Orden mit neuen Pfarrkirchen, denn wenn die Bevölkerungszahl wuchs, ordneten diese einfach mehr Priester zur Seelsorge und Sakramentenspendung ab, ohne das gemäß den strengen Anforderungen des Kirchenrechts neue Benefizien zu fundieren waren. Dem Kirchenrecht ging es dabei um die Sicherung des Einkommens der Pfarrer und anderer Benefiziaten gegenüber Ansprüchen der Gründer und Eigentümer von Kirchen, die sich auf ihre Stifter- und Besetzungsrechte beriefen. Pfarreien wurden deshalb als kirchliche Benefizien definiert mit festen Einkünften. Ohne ausreichende Einkünfte durfte keine Pfarrei und auch keine andere fe-

von ibrer Gründung bis zur Aufhebung, hrsg. v. Karl Hengst, Veröffentlichungen der Historischen Kommission für Westfalen 44/I-3 (Münster: Aschendorff, 1992/2003) für Borken, Burgsteinfurt, Hassel, Herford, Horst, Wietersheim; Nordrheinisches Klosterbuch: Lexikon der Stifte und Klöster bis I8I5, Tl. I, Aachen bis Düren, Tl. 2, Düsseldorf bis Kleve, hrsg. v. Manfred Groten (Siegburg: Franz Schmitt, 2009/20 I 2) für Aachen, Herrenstrunden, Kronenburg, Dinslaken, Düren, Duisburg, Walsum, Jülich; Württembergisches Klosterbuch: Klöster, Stifte und Ordensgemeinschaften von den Anfängen bis in die Gegenwart, hrsg. v. Wolfgang Zimmermann und Nicole Piersching (Ostfildern: Thorbecke, 2003) für Dätzingen, Leonberg, Rohrdorf, Rexingen, Affaltrach, Mergentheim, (Schwäbisch) Hall, Hemmendorf; Hessisches Klosterbuch: Quellenkunde zur Geschichte der im Regierungsbezirk Kassel, im Kreis Grafschaft Schaumburg, der Provinz Oberhessen und dem Kreis Biedenkopf gegründeten Stifter, Klöster und Niederlassungen von geistlichen Genossenschaften, hrsg. v. Wilhelm Dersch, Veröffentlichungen der Historischen Kommission für Hessen und Waldeck I 2 (Marburg: Elwert, I940, 2. Aufl.) für Anzefahr, Butzbach, Kassel, Gelnhausen, Grebenau, Nesselhof, Nidda, Niederweisel, Niederwildungen, Rüdigheim, Wiesenfeld, für Templer Assenheim?, Gelnhausen?, Großen-Linden?, Homburg an der Ohm? 
ste Stelle für einen Messpriester eingerichtet werden. Die Besetzung der Pfarreien und anderen Benefizien wurde den Bischöfen vorbehalten, denen die Inhaber des Patronatsrechts innerhalb bestimmter Frist einen als Priester geeigneten Kandidaten präsentieren mussten. Um Laieneinfluss zurückzudrängen, ermutigte man die Übertragung von Patronatsrechten an geistliche Institutionen. ${ }^{3}$ Davon profitierten nicht zuletzt die geistlichen Ritterorden. Deren Interesse jedoch bestand nicht darin, den Pfarrer oder andere Geistliche für Gottesdienst und Seelsorge ausreichend zu besolden. Vielmehr wollten und sollten sie Überschüsse aus Kirchen und Pfarreien für ihre eigenen Zwecke verwenden, neben den Erträgen des Pfarrgutes selbst insbesondere die Spenden und Oblationen der wachsenden Bevölkerung in Stadt und Land. Das kanonische Recht entwickelte dafür das Rechtsinstitut der Union oder seit Mitte des I 3. Jahrhunderts Inkorporation. Pfarrkirchen wurden Klöstern, Stiften und Orden einverleibt, inkorporiert.

Die genauen Bedingungen der Inkorporation fielen unterschiedlich aus. Gemäß dem hochmittelalterlichen Kirchenrecht kann man zwei Formen unterscheiden, incorporatio non pleno iure und incorporatio pleno iure. ${ }^{4} \mathrm{Im}$ ersten Fall, bei der incorporatio non pleno iure, blieb die Pfarrei als eigene Rechtspersönlichkeit bestehen, mit eigenem Vermögen, nur nicht mehr mit einem Pfarrer, sondern mit einen schlechter bezahlten Pfarrvikar an der Spitze. Wie sonst der Pfarrer, musste jetzt der Pfarrvikar vom Ritterorden als dem Besitzer der Pfarrei der Diözese präsentiert, durch die Diözese geprüft und eingesetzt werden. Der Orden durfte den Pfarrvikar nicht willkürlich versetzen, ohne fristgemäß einen Nachfolger zu präsentieren. Nach außen fiel der Unterschied zwischen Pfarrer und Pfarrvikar deshalb kaum auf, weshalb beide Typen von Geistlichen gewöhnlich einfach als plebanus, pastor, Pfarrherr usw. bezeichnet wurden. Im zweiten Fall, bei der incorporatio pleno iure, verschmolz das Pfarreivermögen dagegen vollständig mit

3 Peter Landau, Ius patronatus. Studien zur Entwicklung des Patronats im Dekretalenrecht und in der Kanonistik des I2. und I3. Jahrhunderts, Forschungen zur kirchlichen Rechtsgeschichte und zum Kirchenrecht I 2 (Köln-Wien: Böhlau, I975); zur Einführung in den heutigen Forschungsstand: Die Pfarrei im späten Mittelalter, hrsg. v. Enno Bünz und Gerhard Fouquet, Vorträge und Forschungen 77 (Ostfildern: Thorbecke, 2013) und die gesammelten Aufsätze Enno Bünz, Die mittelalterliche Pfarrei. Ausgewählte Studien zum I3.-I6. Jahrhundert, Spätmittelalter, Humanismus, Reformation 96 (Tübingen: Mohr Siebeck, 20 1 7 ).

4 Corpus Iuris Canonici, Bd. 2, hrsg. v. Emil Friedberg, Liber Extra, Lib. 5, Tit. 33, Cap. 3 und Lib. 2, Tit. 26, Cap. I 5 (Leipzig: Tauchnitz, I 88 I), Sp. 387-388, 849-850; Thomas Krämer, Dämonen, Prälaten und gottlose Menschen. Konflikte und ihre Beilegung im Umfeld der geistlichen Ritterorden, Vita regularis 64 (Berlin-Münster: LIT, 2015), I08-1 59; die früher mitunter angenommene incorporatio plenissimo iure - vgl. Johannes Baptist Sägmüller, Lebrbuch des katholischen Kirchenrechts, Bd. I (Freiburg im Breisgau: Herder, I 9 I 4, 3. Auflage), 3 I o-3 I I - hat sich nicht nachweisen lassen. 
dem Ordensgut. Den Pfarrvikar musste der Orden nicht mehr der Diözese präsentieren, sondern gegenüber dem Bischof galt rechtlich der Orden selbst als der Pfarrer. In der Pfarrei gab es danach nur einen oder mehrere Geistliche, welche der Orden beliebig ein- und absetzte. Dem Orden allein blieb überlassen, wie er Gottesdienst und Sakramentenspendung organisierte. Zwar galt kirchenrechtlich die Soll-Vorschrift, dem Pfarrvikar eine portio congrua oder canonica zukommen zu lassen. ${ }^{5}$ Was als angemessener Unterhalt zu gelten hatte, wurde freilich von Fall zu Fall recht unterschiedlich ausgelegt. Wenn der Orden gegen die Unterhaltspflicht verstieß, blieb das zunächst eine Sache zwischen dem Orden und der Pfarrbevölkerung; die Diözese konnte erst einschreiten, wenn Mängel bei Gottesdienst und Sakramentenspendung nachzuweisen waren. ${ }^{6}$

Wie bei der incorporatio minus plena blieben jedoch auch bei der incorporatio plena Abgaben und Gerichtsbarkeit der Diözese bestehen. Abgaben mussten entrichtet werden an den jeweils zuständigen Archidiakon, an das jeweils zuständige Landkapitel, an das Domkapitel und an den Bischof. Hinzu kamen Visitation und Gerichtsbarkeit durch den Bischof und seine Untergebenen. Wie andere geistliche Ritterorden beriefen sich die Johanniter dagegen auf ihre vom Papst verliehenen Exemtionsprivilegien. Abgaben sollten sie nicht leisten, weil das die Überschüsse zugunsten des Kampfes gegen die Feinde des Glaubens schmälerte. Die Gerichtsbarkeit über seine Angehörigen wurde vom Papst allein dem Orden selbst vorbehalten. Die praktische Durchsetzung solcher Privilegien jedoch erforderte immer wieder Kompromisse. In der Regel wurden in Mitteleuropa Pfarreien einzelnen Landkapiteln unter einem Land- oder Ruraldekan und darüber einzelnen Archidiakonaten unter einem Archidiakon zugeteilt, der zumeist ein Domherr der Bischofskirche war. Große Stadtpfarreien konnten jedoch von der Aufsicht durch das Landkapitel und den Archidiakon eximiert sein. Nicht überall erhalten, geschweige denn ediert, gab es Listen mit Abgaben und Steuern, welche Pfarreien und Pfründen, darunter auch solche der Johanniter, dem Landdekan, dem Archidiakon oder dem Diözesanbischof selbst entrichten sollten. Die Bischöfe, die sich in Deutschland vornehmlich der weltlichen Regierung ihrer Territorien widmeten, hatten seit der zweiten Hälfte des I 3. Jahrhunderts einen Weihbischof für bischöfliche Weihehandlungen neben sich, dazu einen Generalvikar und einen Offizial für administrativ-jurisdiktionelle Angelegenheiten unter sich.

5 Corpus Iuris Canonici, hrsg. v. Friedberg, Bd. 2: Liber Extra, Lib. I, Tit. 28, Cap. 3 und Lib. 3 , Tit. 5, Cap. I 2, 30, 3 I, Sp. I 56-I 57, 468, 478-479.

6 Wolfgang Petke, "Von der klösterlichen Eigenkirche zur Inkorporation in Lothringen und Nordfrankreich im I I. und I 2. Jahrhundert," Revue d'histoire ecclésiastique 87 (1992): 34-72, 375-404, mit der älteren Literatur. 
Die Übertragung einer Kirche, besonders einer Pfarrkirche, samt dem zugehörigen Besetzungsrecht war ein gewichtiger Rechtsakt mit dauerhafter Folgewirkung. Daher sollte man erwarten, dass stets eine Urkunde ausgefertigt wurde. Allerdings sind nicht für alle Pfarrkirchen im Priorat Alamania solche Urkunden nachzuweisen. Gerade für frühe Erwerbungen wie Bubikon, Duisburg oder Schlawe fehlen sie. ${ }^{7}$ Umgekehrt wurden nicht alle erhaltenen Urkunden dauerhaft in die Realität umgesetzt. So schenkte 1329 Rudolf von Bebenburg dem Johanniterhaus zu Rothenburg die Pfarrkirche in Gammesfeld; er selbst bestätigte dies I 34 I, sein Sohn Engelhard, Domherr zu Würzburg, I $358 .^{8}$ Hintergrund war, dass Rudolf zwei Brüder hatte, Friedrich, der Johanniter und zeitweise Komtur in Rothenburg war, und den durch seine Werke zur Reichspublizistik hervorgetretenen Lupold von Bebenburg, Domherrn und Archidiakon zu Würzburg, später Bischof von Bamberg. Lupold hatte die Pfarrei Gammesfeld vor der Schenkung an die Johanniter selbst besessen. Rudolfs Sohn Engelhard jedoch unterlag im Erbstreit mit zwei Stiefbrüdern Wilhelm und Konrad von Bebenburg, so dass die Pfarrkirche sich spätestens 1367 wieder vollständig in den Händen der ritteradeligen Ortsherrschaft befand. ${ }^{9}$

Der Johanniterorden erhielt Kirchen mit und ohne Seelsorgsrechte. Die Unterscheidung zwischen Pfarrkirchen und bloßen Kapellen war nicht immer einfach. Ordenskirchen, die formal nicht Pfarreien waren, hatten trotzdem öfters Friedhöfe, wo Bewohner des Ordenshauses, Ordensangehörige wie Bedienstete, und Mitglieder der dort bestehenden Bruderschaften sowie andere Wohltäter des Ordens, die alle außerhalb des Ordenshauses lebten, sich beerdigen ließen. Nicht nur für verstorbene Ordensangehörige, sondern auch für Bruderschaftsmitglieder und andere Gönner hielten die Ordensgeistlichen dort Gedächtnisgottesdienste mit Fürbitten. ${ }^{\circ}$ Ebenfalls aufgrund päpstlicher Privilegien durften Bruderschafts-

7 Rödel, Das Großpriorat, 57 (Bubikon), 360 (Duisburg, vgl. dazu jetzt Nordrheinisches Klosterbuch 2: I60; zu Schlawe jetzt Marek Smoliński, "Zur Geschichte der Johanniter und ihrer politischen Rolle in Polen bis zum Jahre 1370," in Regionalität und Transfergeschichte: Ritterordenskommenden der Templer und Johanniter im nordöstlichen Deutschland und in Polen, hrsg. v. Christian Gahlbeck, Heinz-Dieter Heimann und Dirk Schumann, Studien zur brandenburgischen und vergleichenden Landesgeschichte 9, Schriften der Landesgeschichtlichen Vereinigung für die Mark Brandenburg N.F. 4 (Berlin: Lukas, 20 I 4), 8 I.

8 Karl Borchardt, Die geistlichen Institutionen in der Reichsstadt Rothenburg ob der Tauber und dem zugehörigen Landgebiet von den Anfängen bis zur Reformation, Veröffentlichungen der Gesellschaft für fränkische Geschichte IX/37, 2 Tle. (Neustadt/Aisch: Degener, I 988), I 25 Nr. 49.

9 Borchardt, Die geistlichen Institutionen, 370-37 I.

1o Eine in westfälischem Privatbesitz befindliche Handschrift von circa I 445 zeigt auf fol. I 3v und $\mathrm{r} 4 \mathrm{r}$ Ordensgeistliche beim Totenoffizium vor einem offenen Holzsarg, in dem eine als 
mitglieder und Wohltäter dort den Gottesdienst besuchen und die Sakramente empfangen, sogar wenn ein Interdikt verhängt worden war, das andernorts in der betroffenen Region dies untersagte.

Pfarreien waren überdies nicht die einzigen Pfründen, über die der Orden verfügte. Erwähnt werden müssen auch Messpfründen, seien es Frühmessen in Landkirchen oder Ewigmessen in Stadtkirchen. Solche Messpfründen wurden gestiftet, teils für Ordens-, teils auch für Weltgeistliche, aber mit Präsentations- oder wenigstens Nominationsrecht für den Johanniterorden. Sie waren gedacht für jeweils einen Priester, der an einem bestimmten Altar mehrfach in der Woche eine Messe halten und für die Stifter beten musste. Gerade im Spätmittelalter nahmen solche Stiftungen einen großen Aufschwung. Wenn die Messpfründe innerhalb einer dem Orden gehörenden Pfarrei entstand, bekam der Orden in der Regel das Präsentationsrecht. Oft jedoch behielten sich die Stifter, ihre Nachfahren oder in ihrem Auftrag der Stadtrat ein Nominationsrecht vor, was bedeutete, dass der Orden einen Geistlichen dem Diözesanbischof vorschlagen musste, den andere auswählten. Beispiele dafür gibt es zahlreich; bekannt sind in der zweiten Hälfte des I 4. Jahrhunderts die unter anderem über dieses Thema geführten Streitigkeiten zwischen der Stadt Bahn und der Kommende Rörchen / Wildenbruch in Pommern. ${ }^{\text {II }}$

Messpfründen unterlagen wie Pfarreien der Aufsicht durch die Diözese und sind deshalb zu unterscheiden von Stellen für einen zusätzlichen Ordenspriester in einem Ordenskonvent, wie sie gelegentlich ordensintern fundiert wurden. Graf Berthold von Henneberg beispielsweise, Prior von Böhmen und Polen im Exil, stiftete 1329 eine zweite Priesterstelle im Ordenshaus Biebelried und gleichzeitig, aber in separater Urkunde eine dritte Priesterstelle im Ordenshaus Reichardsroth. Falls der Orden dem nicht nachkam, sollte das Stiftungsgut für die Stelle in Biebelried an Dekan und Kapitel des Stifts Haug in Würzburg, für die Stelle in Reichardsroth an das unweit gelegene Zisterzienserinnenkloster Frauental fal-

Johanniter gekleidete Person liegt: Jochen Luckhardt, "Zur Bildausstattung des Totenbuches der Johanniterkommende Burgsteinfurt," Masters and Miniatures. Proceedings of the Congress on Medieval Manuscript Illumination in the Northern Netherlands (Utrecht, Io-I3 December 1989), hrsg. v. Koert van der Horst und Johann-Christian Klamt, Studies and Facsimiles of Netherlandish Illuminated Manuscripts 3 (Doornspijk: Davaco, I99 I), I95-200, mit 2 Abb.

I Rödel, Das Großpriorat, 434, 440-44 I. 
len. ${ }^{12}$ Ähnlich wurde für den Konvent in Köln 1382 eine achte und I 480 eine neunte Stelle für einen Ordenspriester geschaffen. ${ }^{13}$

Eigentlich durften Ordensangehörige nach ihrem Ordenseintritt über kein Privatvermögen mehr verfügen, um solche Stiftungen für ihr persönliches Seelenheil durchzuführen. Aber darüber sah man je länger, desto mehr hinweg, insbesondere bei einflussreichen und verdienten Ritterbrüdern. Bis zum i 5 . Jahrhundert setzte es sich durch, dass Ordensbrüder mit ihrem persönlichen Nachlass beliebig stiften durften, darunter auch Messpfründen. Mit goldenem und silbernen Geschirr, wertvollem Bettzeug, 700 Gulden Bargeld und 600 Maltern Getreide fundierte Fr. Georg von Scharenstetten, bis zu seinem Tode Statthalter des Ordenshauses Reichardsroth, zwei Messpfründen in der markgräflich-ansbachischen Amtsstadt Crailsheim; ob zur Verleihung an Ordens- oder Weltgeistliche, bleibt offen, weil die eigentliche Fundationsurkunde bisher nicht aufgetaucht ist. Das Präsentationsrecht sollte dem Komtur von Rothenburg zustehen. Dagegen protestierte der Komtur von (Klein)Erdlingen, weil Fr. Georg von Scharenstetten nie regulär Komtur von Reichardsroth und Rothenburg gewesen und außerdem zu (Klein) Erdlingen in den Johanniterorden eingetreten sei, weshalb sein Nachlass diesem Hause zustünde. Zwei Ordenskapitel des Priorats Alamania zu Speyer I 464 und zu Basel 1466 entschieden jedoch zugunsten von Rothenburg, weil Georg bis zu seinem Tode als Statthalter von Reichardsroth sowie als Kommendeverwalter, wenn auch nicht formell als Komtur in Rothenburg amtiert und das Fundationsgut in dieser Amtszeit erwirtschaftet habe. ${ }^{14}$ Die drei im Anhang edierten Dokumente, ein Notariatsinstrument und zwei auf dem Provinzialkapitel ausgestellte Urkunden, beleuchten beispielhaft rechtlich und administrativ komplexe, schwer zu bewältigende Problematiken innerhalb des Ordens zwischen Armutsgelübde

I2 I 329 August I 5: Borchardt, Die geistlichen Institutionen, 672, Nr. 76; sollte in Biebelried zwei Monate hintereinander kein zweiter Priesterbruder leben, fiel der Zins für das laufende Jahr an Dekan und Kapitel des Stifts Haug. Stellte der Komtur in Biebelried im folgenden Jahr wieder einen zweiten Priesterbruder an, dann erhält die Kommende auch diesen Zins wieder. Berthold verstarb am 2 I. August I 330 : Die Würzburger Inschriften bis I525, hrsg. v. Karl Borchardt und Franz Xaver Herrmann, Die Deutschen Inschriften 27 (Wiesbaden: Reichert, I 988), 36 Nr. 54.

13 Sang-Joon Ahn, Die Kölner Johanniterkommende Sankt Johann und Cordula im Spätmittelalter: Geschichte, Besitz, Wirtschaft und Sozialstruktur, Kölner Schriften zu Geschichte und Kultur 28 (Köln: SH-Verlag, 2006) i I 6- I 7.

14 Borchardt, Die geistlichen Institutionen, I 37-I38; Ausfertigungen München, Bayerisches Hauptstaatsarchiv, Ritterorden Urkunden [künftig: BayHStAM RiU], 242, 243, 244, ediert im Anhang Nr. I-3; dazu ausführlicher Karl Borchardt, "Hospitaller Chapters in the Medieval Priory of Alamania," in Piety, Pugnacity and Property, hrsg. v. Nicholas Morton, The Military Orders 7 (London: Routledge, 2019), I95-207. 
und Stifterwillen des Fr. Georg von Scharenstetten, aber auch im Verhältnis von Orden und Weltkirche in der Stadt Crailsheim.

Statt der vorgesehenen zwei Pfründen reichte das Gut jedoch nur für eine Ewigmesse im Spital zu Crailsheim. Das Besetzungsrecht für diese Ewigmesse übte die Kommende Rothenburg. Deren Statthalter Fr. Burkhard Megel präsentierte darauf ${ }_{4} 497$ den Johanniter Fr. Michael Karpf. ${ }^{15}$ Da Georg von Scharenstetten nach dem Notariatsinstrument von 1464 Bettzeug und Hausrat in Crailsheim hinterlassen hatte, wo es kein Ordenshaus gab, und auch in Crailsheim verstorben war, könnte er dort gewissermaßen "privat" gewohnt haben. Jedenfalls hatte der Pfründenstifter persönliche Beziehungen zu Crailsheim und dessen Umgebung. Auf sein Betreiben hin kaufte nämlich die Kommende Rothenburg I 46 I um 409 fl das Patronatsrecht der nahegelegenen Pfarrei Ellrichshausen vom Dominikanerinnenkloster in Rothenburg, ${ }^{16}$ genau an dem Ort, wo ein Viertel des Zehnten und weitere Gefälle die beiden Messpfründen finanzieren sollten. Nach dem Verzicht des seit 1457 amtierenden Pfarrers Markus Pfingstag präsentierte Fr. Burkhard Megel als Statthalter der Kommende Rothenburg I 499 auf dem Pfarrei Ellrichshausen den Säkularpriester Ludwig Hemmendorfer. Doch schon I 5 I 4 nahm Markgraf Friedrich IV. von Ansbach als Landesherr das Präsentationsrecht wahr. ${ }^{17}$ Dass eine Johanniterkommende Geld anlegte, indem sie eine Patronatspfarrei kaufte, war eher ungewöhnlich. Der Kauf I 46I in Ellrichshausen wird deshalb bereits mit der geplanten Stiftung durch Fr. Georg von Scharenstetten in Crailsheim zusammenhängen.

is Regest von Paul Schöffel aus den I 945 verbrannten bischöflichen Registern Würzburg, ı o. Mai I 497, Diözesanarchiv [künftig: DAW], S I, fol. I 2 r.

16 Priorin Elisabeth Zwinger, Komtur Georg von Scharenstetten: Beschreibung des Oberamts Crailsheim, hrsg. von. K. statistisch-topographisches Bureau (Stuttgart: Kohlhammer, I 884), 253; Borchardt, Die geistlichen Institutionen, I 28, I 76; vorbesitzer des Patronatsrechts war der Ritteradelige (Hoch)Brand von Seinsheim. Ellrichshausen lag im Archidiakonat VI, Landkapitel Crailsheim: Franz Josef Bendel, "Die Würzburger Diözesanmatrikel aus der Mitte des I 5. Jahrhunderts," Würzburger Diözesangeschichtsblätter 2/2 (1 934), 9 Nr. 34 I.

17 Präsentation des Markus Pfingstag, 31. Oktober 1457, Ausfertigung BayHStAM RiU, 238; Investitur, 2. November 1457: ebd. 239; präsentation des Ludwig Hemmendorfer, 26. Juni I 499, Regest DAW, S I, fol. I 4v; Investitur, 28. Juni I 499, Ausfertigung BayHStAM RiU, 253. Ludwig Hemmendorfer war 1483 Spitalschreiber und bei seinem Tode I 5 I 0 Kaplan am Allerheiligenaltar im Spital zu Rothenburg: Borchardt, Die geistlichen Institutionen, 60 I, Nr. 477; Präsentation durch Markgraf Friedrich IV., 5. Mai I 5 I 4, Regest DAW, S I, fol. I 4V; Borchardt, Die geistlichen Institutionen, I 42; Beschreibung des Oberamts Crailsheim, hrsg. von K. statistisch-topographisches Bureau, 253: Albrecht Klingler, sieben Jahre am markgräflichen Hof, I 5 I0-I 5 I 3 Pfarrverweser zu Crailsheim, nach dem Tode des Martin Rosler I 5 I 6- I 544 Pfarrer zu Ellrichshausen, Dekan des Landkapitels, zur Reformation übergetreten, erwarb das Patronat in Ellrichshausen für den Markgrafen. 


\section{Administrative Strukturen}

Mit den Stichworten Pfarreien, Kapellen, Messpfründen, Patronat und Inkorporation sowie Präsentation und Nomination ist der Hintergrund umschrieben, um einerseits die Rolle der Geistlichen im Johanniterpriorat Alamania, andererseits die Bedeutung der Johanniter in den Diözesen zu untersuchen. Eine klare Statistik erlaubt der Forschungsstand derzeit nicht. Überdies sprechen Zahlen erst, wenn man sie mit anderen Gebieten des lateinischen Europa vergleichen kann, was angesichts des Forschungsstandes erst recht illusorisch erscheint. Neben Quellen aus den Archiven der Johanniter selbst wären spätmittelalterliche Diözesanverzeichnisse heranzuziehen, beispielsweise die Investiturprotokolle und Annatenregister des Bistums Konstanz ${ }^{18}$ oder die Diözesanmatrikel von Würzburg. ${ }^{19}$ Investiturprotokolle sind vor allem deshalb bedeutsam, weil Urkunden im Zusammenhang mit Präsentationen naturgemäß nur für den Präsentierten bedeutsam waren und nach dessen Ableben vielfach makuliert wurden, so dass die Ausfertigungen nicht mehr erhalten sind, sondern allein die Registereinträge. So präsentierte für die Pfarrkirche in Bubikon der Prokurator des dortigen Johanniterhauses nach dem Tode des Fr. Michael Äblin I 465 den Fr. Rudolf Eggenberg; nach dessen Tod präsentierte der Prior von Alamania Fr. Graf Rudolf von Werdenberg 1483 höchstpersönlich den Fr. Andreas Gubelmann, und diesem wurde 1492 durch den Bischof erlaubt, für ein Jahr von der Pfarrei Bubikon abwesend zu sein. ${ }^{20}$ Auch anderes geht mitunter aus solchen Protokollen hervor: So befehdeten einander 1436 die Johanniter und die Stadt Freiburg; für die Pfarrkirche in Heitersheim wurde deshalb den Johannitern erlaubt, dort befristet und ausnahmsweise einen Weltpriester einzusetzen. ${ }^{21}$ Warum die Fehde den Orden daran hinderte, einen Ordensgeistlichen zu präsentieren, bleibt freilich im Dunkeln.

Annaten waren die Früchte eines Benefiziums im ersten Jahr nach Eintritt einer Vakanz. Sie standen dem Bischof, ab einem bestimmten Pfründenwert bei Besetzung durch den Papst der apostolischen Kammer zu. Geistliche Institutionen, denen

18 Manfred Krebs, "Die Investiturprotokolle der Diözese Konstanz aus dem I 5. Jahrhundert," Freiburger Diözesan-Archiv 66 (1938): Anhang I-104; bis 73 (1953): Anhang 787-1047; ders., "Die Annaten-Register des Bistums Konstanz aus dem I 5. Jahrhundert," Freiburger Diözesanarchiv 76 (1956): I-467; dazu ders., "Die Annatenregister des Bistums Konstanz und ihre Bedeutung für die württembergische Kirchengeschichte," Zeitschrift für württembergische Landesgeschichte I 3 (1954): 109-I I 9.

19 Bendel, "Die Würzburger Diözesanmatrikel," I-XXX, I-43.

20 Krebs, "Die Investiturprotokolle," I 26.

${ }_{21}$ Ibid., 367. 
Pfarreien und andere Benefizien inkorporiert waren, protestierten dagegen, sogar in der Diözese Konstanz, wo der Bischof traditionell nur die Hälfte der Früchte des ersten Vakanzjahres verlangte. Nach Diskussionen unter anderem auf dem Konzil zu Konstanz erwirkten die geistlichen Ritterorden im Bistum Konstanz I 4 I 7 / 420 einen Kompromiss. 7 I Kirchen der Johanniter ${ }^{22}$ und 46 Kirchen des Deutschordens, ${ }^{23}$ die alle vor 1378 inkorporiert worden waren, wurden von der Annatenpflicht befreit; erhalten blieb die Annatenpflicht nur für nach I 378 bereits inkorporierte oder künftig zu inkorporierenden Pfarreien, bei den Johannitern für Gündlingen (Dekanat Breisach), Dübendorf (Dekanat Zürich), Wengi (Dekanat Frauenfeld) und Wuppenau (Dekanat Wil), beim Deutschorden für Hohenberg, Münsingen, Rüti bei Bern und Rottelfingen. ${ }^{24}$ Demgemäß zahlte Fr. Andreas Hammermeister I 493 für Gündlingen 50 fl, der Komtur von Tobel in den soer Jahren des I 5 . Jahrhunderts und der Pfarrvikar I 505 für Wengi jeweils $36 \mathrm{fl}$ an den Bischof. ${ }^{25}$

22 (I) Dekanat Freiburg: Vörstetten, Altenkrenzingen; (2) Dekanat Endingen: Achkarren, Bikkensohl, Oberbahlingen; (3) Dekanat Breisach: Kirchzarten, Oberrimsingen, Weinstetten, Schlatt, Bremgarten; (4) Dekanat Neuenburg: Grißheim, Heitersheim, Britzingen, Riedlingen, Steinenstadt, Au, Schliengen; (5) Dekanat Wiesental: Warmbach; (6) Dekanat Villingen: Pfohren, Dürrheim, Grüningen, Weigheim; (6) Dekanat Stühlingen: Lenzkirch; (7) Dekanat Rottweil: Obereschach, Neuhausen, Dorf Villingen; (8) Dekanat Haigerloch: Isingen mit der filia Rosenfeld; (9) Dekanat Horb: Altheim, Rexingen; ( Iо) Dekanat Herrenberg: Unterjettingen, Ebhausen mit der filia Rohrdorf; ( I I) Dekanat Tübingen: Dettingen, Hemmendorf; (1 2) Dekanat Reutlingen: Betzingen, Oferdingen; ( 13 ) Dekanat Trossingen: Aldingen, Nendingen; ( 14 ) Dekanat Munderkingen: Oberstadion; ( I 5 ) Dekanat Wil: Tobel, Weilen, Affeltrangen, Märwil; ( I 6) Wetzikon: Hinwyl, Wald, Wangen, Bubikon; ( 17 ) Dekanat Regensberg: Buchs; ( I 8) Dekanat Zürich: Horgen für die Hälfte der Früchte, Wädenswil, Richterswil, Küssnacht; (19) Dekanat Syns oder Hochdorf: Hohenrain, Dietwil, Aesch, Muswangen, Römerswil; (20) Dekanat Büren: Wohlen, Aettingen; (2 I ) Dekanat Münsingen: Buchsee, Seedorf, Burg Bremgarten.

23 ( I) Kommende Ulm: Horningen, Lautern, Dormettingen, Ebersbach; (2) Kommende Winnenden bei Esslingen: Winnenden; (3) Kommende Freiburg: Malterdingen, Buchheim, Kappel, Wasenweiler, Merdingen, Ballrechten, Hausen, Denzlingen, Bötzuingen, Ihringen; (4) Kommende Beuggen: Hertingen (Dekanat Neuenburg), Beuggen genannt Oberkirch, Rickenbach, Herthen, Wilen, Hasel, Nollingen (Dekanat Wiesental), Birndorf (Dekanat Waldshut), Lengnau (Dekanat) Regensberg); ( 5 ) Kommende Bern: Rüderswil, Rüti bei Burgdorf; Sumiswald: Dorf Sumiswald, Roth, Affoltern, Trachselwald; (6) Kommende Hitzkirch: Dorf Hitzkirch, Menznau mit Filia Hasli, Altishofen; (7) Kommende Mainau: Ittenhausen, Pfaffenhofen, Lippertsreutte, Überlingen, Rast, Mindersdorf, Dingelsfort; (8) Kommende Altshausen: Altshausen, Opfenbach, Winnenden, Ebersach, Fleischwangen, Esenhausen.

24 Konstanz 26. August I 417, Konstanz 21. August I 420, Konstanz I 5. November I 420 : Regesta episcoporum Constantiensium, Bd. 3, ${ }_{3} 84-1436$, hrsg. v. Karl Rieder (Innsbruck: Wagner, I 926 ), Nr. 8567, 8793, 88 I 3.

25 Manfred Krebs, "Die Annatenregister des Bistums Konstanz und ihre Bedeutung für die württembergische Kirchengeschichte," Zeitschrift für württembergische Landesgeschichte I 3 
Im Prinzip könnte man auf solche Weise aus der Überlieferung der Diözesen und der Johanniter das flächendeckende Pfarrnetz sowie den Anteil der Johanniter daran rekonstruieren. Der war deutlich kleiner als bei alten Benediktinerklöstern oder Kollegiatstiften. Sinnvoll vergleichen ließen sich Zisterzienser und Prämonstratenser, ebenfalls Orden des I 2. Jahrhunderts. Zahlenmäßig dürften sich die Johanniter des Reichsgebiets nördlich der Alpen in einer ähnlichen Größenordnung bewegt haben wie der Deutsche Orden; die Templer dagegen waren in Mitteleuropa deutlich geringer vertreten, wenn auch mutmaßlich in ganz ähnlichen Strukturen. Die Bettelorden des I 3. Jahrhunderts, besonders die Dominikaner und Franziskaner, übernahmen keine Pfarreien mehr. Geographisch reichte das Johanniterpriorat Alamania vom Hochrhein bis in die Niederlande und bis zur Weichselmündung. Seine Ordenshäuser und -kommenden formierten acht Balleien, ( I) Utrecht, (2) Westfalen, (3) Sachsen, die Mark Brandenburg, das Wendland und Pommern, (4) Thüringen, (5) Franken, (6) die Wetterau, ${ }^{26}(7)$ Köln und (8) das Oberland, gemeint ist das Gebiet beiderseits des Oberrheins. Sie lagen in acht Kirchenprovinzen, von Besançon über Mainz, Trier und Köln, Salzburg, Hamburg-Bremen und Magdeburg bis Gnesen und knapp 40 Diözesen.

\section{Ordenshäuser, Kapellen und Pfarrkirchen}

Eine Reihe von Ordenshäusern (domus, auch curie) bestanden neben Pfarrkirchen in Dörfern, welche dem Johanniterorden geschenkt oder von ihm gekauft worden waren. ${ }^{27}$ Teils waren Ordenskirche und Pfarrkirche identisch, beispielsweise in Duisburg und Mergentheim; teils hatten die Johanniter die Pfarrkirche erst nach dem Ausstattungsgut ihrer Niederlassung erworben, so dass die Kirche für das Volk zwar am gleichen Ort, aber entfernt von der Ordensniederlassung situiert war, beispielsweise in Steinfurt, wo man zwischen der Großen und der Kleinen

(1954): I 50, I 8 I, I 83.

26 Karl Borchardt, "Tätigkeit und Karrieren von Johannitern in der Ballei Wetterau," in Johanniter in Hessen. 800 Jahre diakonischer Auftrag, hrsg. v. Reiner Braun und Ruth Slenczka, Jahrbuch der Hessischen Kirchengeschichtlichen Vereinigung 59 (Darmstadt: Hess. Kirchengeschichtl. Vereinigung, 2008), 17-33.

27 Rödel, Das Großpriorat, 57, 60 (Bubikon), 68 (Tobel), 86 (Leuggern), 92 (Küsnacht), ı I (Buchsee), 333 (Thunstetten), 99 (Reiden), 130 (Rexingen), I 37 (Dätzingen), 253 (Sulzbach), 268 (Rüdigheim), 272 (Nidda), 294 (Mossau, Pfarrkirche 1253 bestätigt durch Erzbischof Gerhard von Mainz, Cartulaire général de l'Ordre des Hospitaliers de S. Jean de Jérusalem, Bd. 2, hrsg. v. Joseph Delaville le Roulx (Paris: Leroux, I 897), 747 Nr. 2656), 299 (Roth an der Our), 302-303 (Adenau), 354 (Burg an der Wupper), 364 (Walsum), 38 I (Steinfurt). 
Kirche unterschied.$^{28}$ Manche Dörfer wurden zu Städten, nachdem die Johanniter die Pfarrkirche übernommen hatten, beispielsweise Mergentheim, Meisenheim oder Feldkirch, ${ }^{29}$ manche auch nur zu Städtchen wie Heitersheim, wo seit dem I 6. Jahrhundert der Prior von Alamania gewöhnlich residierte..$^{30}$ Dass die Johanniter eine Stadtpfarrkirche übernahmen, war dagegen im Altsiedelland sehr selten. Hagenau ist ein Beispiel. ${ }^{31}$ Häufiger kam dies nur in den ostmitteleuropäischen Ausbaugebieten vor; im Priorat Alamania wären zu nennen Freyenstein und Lychen im Grenzgebiet zwischen Brandenburg und Mecklenburg sowie Königsberg/ /Neumark, Stargard an der Ihna, Bahn und Arnswalde in der brandenburgischen Neumark und dem angrenzenden Pommern. ${ }^{32}$

Bevor die Johanniter eine Pfarrkirche übernahmen, amtierten dort in der Regel Weltgeistliche, die dem zuständigen Bischof vom Patronatsherrn präsentiert wurden. Der Patronatsherr war rechtlich verpflichtet, einen Mann vorzuschlagen, der entweder schon Priester war oder alle Voraussetzungen erfüllte, um die Priesterweihe binnen bestimmter Frist zu empfangen. Wenn sich der Patronatsherr daran hielt, war der zuständige Bischof seinerseits verpflichtet, den Vorschlag des Patronatsherrn anzunehmen. Nach der Investitur folgte dann die körperliche Einweisung in den Besitz der Pfarrkirche und der zugehörigen Einkünfte. Diese

28 Rödel, Das Großpriorat, I 62, 362, 382. Zu Steinfurt Marc Sgonina, Die Johanniterballei Westfalen: Unter besonderer Berücksichtigung der Lebensformen der Zentralkommende Steinfurt und ibrer Membra (Frankfurt/Main: Lang, 20 I 4), passim.

29 Rödel, Das Großpriorat, I 58 (Mergentheim), 249 (Meisenheim), 73 (Feldkirch).

30 Ibid., 322-323 (Heitersheim).

31 Ibid., 22 I; Patronat von St. Georg 1262 November 3 durch König Richard von Cornwall, Cartulaire général de l'Ordre des Hospitaliers de S. Jean de Jérusalem, Bd. 3, hrsg. v. Joseph Delaville le Roulx (Paris: Leroux, I 899), 54-55 Nr. 304I; Cartulaire de l'Église S. George de Haguenau, hrsg. v. Charles Auguste Hanauer, Quellenschriften der Elsässischen Kirchengeschichte 5 (Straßburg: Le Roux, I 898), i I-I 2 Nr. i 8. Die Zustimmung der Stadt erkauften sich die Johanniter 1359 für 5000 Gulden mit einem Jahreszins von 300 Gulden an die Kirchenfabrik: ebd., 89-94 Nr. I 5 I.

32 Rödel, Das Großpriorat, 434, 440-44 I; Christian Gahlbeck, "Strukturreform um der Wirtschaftlichkeit willen? Die Ballei Brandenburg der Johanniter unter Hermann von Warberg nach I 350 ," Jahrbuch für die Geschichte Mittel- und Ostdeutschlands 62 (2016): I 23-I 24; Rainer Szczesiak und Christian Gahlbeck, "Die Kommenden der Johanniter in Mirow, Gardow und Nemerow in der Herrschaft Stargard in Südmecklenburg," Regionalität und Transfergeschichte: Ritterordenskommenden der Templer und Johanniter im nordöstlichen Deutschland und in Polen, hrsg. v. Christian Gahlbeck, Heinz-Dieter Heimann und Dirk Schumann, Studien zur brandenburgischen und vergleichenden Landesgeschichte 9, Schriften der Landesgeschichtlichen Vereinigung für die Mark Brandenburg N.F. 4 (Berlin: Lukas, 20 I 4), 204-247; Agnieszka Lindenhayn-Fiedorowicz, "Johannitisches Patronat und städtische Architektur: Die Marienkirche zu Stargard (Stargard Szczeciński) in Pommerns," in Regionalität und Transfergeschichte, hrsg. v. Gahlbeck, Heimann und Schumann, 248-270. 
Kollation fand nicht in der Bischofsstadt, sondern am Ort der Pfarrkirche statt. Vorgenommen wurde sie durch Beauftragte des Bischofs. Fast überall im deutschen Johanniterpriorat waren die Diözesen in Archidiakonate eingeteilt. Deshalb war es in der Regel die Aufgabe des Archidiakons, gewöhnlich eines Domherrn, die körperliche Besitzeinweisung vornehmen zu lassen. Vier Urkunden wurden ausgestellt, die Präsentation durch den Patronatsherrn an den Bischof, die Investitur durch den Bischof für den neuen Pfarrer, die Kollation oder körperliche Besitzeinweisung durch den Archidiakon für den neuen Pfarrer und vielfach viertens ein Versprechen des neuen Pfarrers, seine Pflichten getreulich zu erfüllen; Empfänger der letzteren Urkunde war gewöhnlich nicht der Bischof oder der Archidiakon, sondern der Patronatsherr, bei Pfarrkirchen unter dem Patronat der Johanniter also der Orden. ${ }^{33}$ Der neue Pfarrer konnte Ordensgeistlicher sein, aber auch Weltgeistlicher. Seinem Unterhalt diente das Ausstattungsgut der Pfarrei. Wenn die Pfarrkirche dem Orden pleno iure inkorporiert war, entfielen Präsentation, Investitur und Kollation, weil in diesem Falle die Johanniter gewissermaßen als ständiger Pfarrer galten, eine Vakanz und Neubesetzung gegenüber dem Diözesanbischof also nicht erfolgte. Folglich erschienen die sehr wohl mit Pfarrechten ausgestatteten Gotteshäuser in Biebelried und Reichardsroth nicht in der Mitte des I 5. Jahrhunderts zusammengestellten Würzburger Diözesanmatrikel. ${ }^{34}$ Anderswo wird das ähnlich gewesen sein.

Die meisten Ordenshäuser im Priorat Alamania jedoch bestanden nicht bei einer Pfarrkirche. Vielmehr enthielten sie nur eine meist Johannes dem Täufer geweihte Kapelle oder hatten neben sich eine eigene Kapelle St. Johannes Baptista. ${ }^{35}$ Nur ausnahmsweise handelte es sich um eine Kapelle, die bereits bestand, als sie den Johannitern geschenkt oder von ihnen erworben wurde, ablesbar meist an dem Patrozinium, das die Johanniter beibehielten, z. B. St. Ludgeri in Borken oder St. Leonhard in Regensburg. ${ }^{36}$ Solche Kapellen in oder bei Ordenshäusern dienten dem Gottesdienst und dem Sakramentenempfang der in dem Ordenshaus le-

33 Ahn, Die Kölner Johanniterkommende, I 25- I 26 zu den Pfarrern der dem Kölner Haus I 36 I inkorporierten Pfarrei Lövenich.

34 Bendel, "Die Würzburger Diözesanmatrikel," I-XXX, I-43.

35 Rödel, Das Großpriorat, 64 (Wädenswil), 76 (Basel), 8 I (Rheinfelden), 90 (Biberstein), 94-95

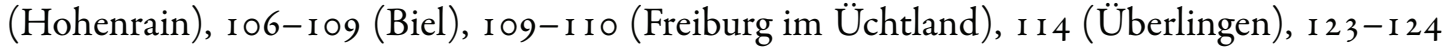
(Rottweil), 3 I 8 (Freiburg im Breisgau), I 39- I 40 (Hall am Kocher), I 49 (Erdlingen), I 54 (Rothenburg ob der Tauber), I72 (Würzburg), I 8 I (Straßburg), I93 (Schlettstadt), I 99 (Sulz), 206 (Mülhausen), 232-233 (Speyer), 242-243 (Worms), 236 (Bruchsal), 255 (Mainz), 263 (Frankfurt am Main), 296 (Trier), 309 (Köln), 344 (Breisig), 35 I (Aachen), 349 (Mechelen), 374 (Herford).

36 Rödel, Das Großpriorat, 377 (Borken), I64 (Regensburg). Die Kirchen der Johanniter hatten Altäre mit höchst unterschiedlichen Patrozinien; im Regelfall aber waren St. Johannes der Täu- 
benden Ordensbrüder und ihrer Bediensteten. Dieser Personenkreis konnte dort auch begraben werden, entweder in oder bei der Kapelle. Allgemeine Pfarrechte aber besaßen diese Kapellen nicht. Wieweit Wohltäter des Ordens dort an Gottesdiensten teilnehmen, Sakramente empfangen und begraben werden durften, war eine Streitfrage. Die päpstlichen Privilegien des I2. und I3. Jahrhunderts sahen das in der Regel vor, trafen aber auf den hartnäckigen Widerstand des Pfarrklerus und der hinter diesem stehenden Bischöfe.

\section{Patronat und Inkorporation von Pfarreien}

Gleichgültig, ob das Ordenshaus nur eine Kapelle hatte oder bei einer Pfarrkirche lag, so verfügten etliche Ordenshäuser über eine oder mehrere Pfarrkirchen in einer Stadt oder in Dörfern, bei denen der Orden das Patronatsrecht besaß oder die dem Orden sogar inkorporiert worden waren. Mehr als eine Pfarrkirche außerhalb des Hauses unterstand den Johannitern bei immerhin 20 Ordenshäusern, von Bubikon, ${ }^{37}$ Tobel, ${ }^{38}$ Feldkirch, ${ }^{39}$ Buchsee, ${ }^{40}$ Überlingen, ${ }^{41}$ Villingen,${ }^{42}$ Rexingen ${ }^{43}$

fer, Maria und Heiligkreuz vertreten: ibid., 60 (Bubikon), 74 (Feldkirch), 84 (Rheinfelden), 96 (Hohenrain), I I 6 (Überlingen), I 2 I (Villingen), I 28 (Hemmendorf), I 34 (Rohrdorf) usw.

37 Ibid., 6r: 5 Pfarrkirchen in Bubikon selbst, Hienwil, Wald, Buchs und Wangen.

38 Ibid., 69-70: 7 Pfarrkirchen in Tobel selbst, Bussnang, Wengen, Wuppenau, Wil, Affeltrangen, Märwil.

39 Ibid., 77: 4 Pfarrkirchen in Feldkirch selbst, Thüringen, Ludesch, Nenzing

40 Ibid., I 02: 6 Pfarrkirchen Buchsee selbst, Twann I 252 (Diözese Lausanne), Moosseedorf I 256 , Krauchthal I 274-I 37 I, Bremgarten/Aare I 307, Wohlen/Aare I 32 I (alle Diözese Konstanz).

4I Ibid., I I 4, I I 6: 3 Pfarrkirchen Goldbach I 260 im Tausch gegen Hagenau: Cartulaire general, 2, hrsg. Dellavile le Roulx, 885 Nr. 2944, Hoppetenzell und Andelshofen. Überlingen selbst zählte zur Pfarrei Aufkirch, welche 1343 dem Deutschordenshaus auf der Mainau inkorporiert wurde; darauf verselbständigte man Überlingen zu einer separaten Pfarrei.

42 Rödel, Das Großpriorat, I 2 I: 9 Pfarrkirchen Dürrheim, Pforheim, Obereschach, Weigheim, Lendingen, Neuenhausen, Lenzkirch, Neuenburg, Grüningen.

43 Ibid., I30-13 I. Rexingen selbst I275, Dettingen I3 19 gegen Burg Rohrau eingetauscht, Altheim I 327, Pfalzgrafenweiler, Schwalldorf I 437, Ergenzingen I 6 I 9. 
Rohrdorf, ${ }^{44}$ (Schwäbisch) Hall,,55 (Altmühl)Münster, ${ }^{46}$ Straßburg, ${ }^{47}$ Sulz, ${ }^{48}$ Freiburg im Breisgau, ${ }^{49}$ Thunstetten, $^{50}$ Heimbach, ${ }^{51}$ Nidda, ${ }^{52}$ (Nieder)Weisel, ${ }^{53}$ Wildungen, ${ }^{54}$ Adenau ${ }^{55}$ bis Haarlem. ${ }^{56}$

Im I 4. Jahrhundert kam es noch vor, dass in einem Konvent mehr ungeweihte Religiosen, fratres laici, lebten als Ordensgeistliche, vor allem im Nordosten, in Mirow, Nemerow, Lietzen, Quartschen, Lagow, Rörchen, Zachan und Tempelburg, aber auch in Boxberg, Kühndorf, (Nieder)Weisel, Heimbach, Dorlisheim, Hemmendorf, Rottweil, Überlingen, Tobel, Bubikon, Hohenrain, Buchsee. Die Soll-Zahlen für das Priorat Alamania sahen 1367 insgesamt 366 Ordensgeistliche und 358 fratres laici vor. Bis zum Ende des I 5 . Jahrhunderts änderte sich das grundlegend: Immer weniger milites verwalteten den Ordensbesitz, damit der einzelne Ritterbruder immer mehr persönliche Einkünfte zur Verfügung hatte. Die Zahl der Geistlichen jedoch blieb unverändert, stieg sogar leicht an, wegen ihrer geistlichen Pflichten und neuer Stiftungen. Bei der Visitation von I 494/ I 495 standen

44 Ibid., I 35: Io Pfarrkirchen Ebhausen, Zwerenberg, Unternotingen, Hochdorf, Ofterdingen, Ilsfeld, Beilstein, Wüntzerhausen, Kaltenwesten, Gündringen/Gondergingen.

45 Ibid., I 44: St. Johann in Hall selbst, früher abhängig von Gottwollshausen (Archidiakonat VI, Kapitel Hall), das dem Orden inkorporiert war, mit Kapellen in Heimbach und dem Weiler ronden. Pfarreien Affaltrach (Archidiakonat VII, Kapitel Weinsberg) und Eschenthal (Archidiakonat VI, Kapitel Künzelsau). Frühmesse Dörzbach (ebenso). Dazu Bendel, "Die Würzburger Diözesanmatrikel," I I Nr. 462; I 2 Nr. 502; I O Nr. 4 I 2; I O Nr. 397.

46 Rödel, Das Großpriorat, I70: 7 Pfarrkirchen Altmühlmünster selbst, Wolfsbuch, Schwainkofen, Harlanden, Theißing, Ernersdorf, Mühlbach.

47 Ibid., I 86: 4 Pfarrkirchen Westhofen, Beelenheim, Huckswire?, Osthausen.

48 Ibid., 20 I : 3 Pfarrkirchen Friesen I 284, Münchhausen, Largitzen, Kapelle Zell.

49 Ibid., 320 : I 3 Pfarrkirchen in Kirchzarten, Britzingen, Grissheim, Schlatt, Oberrimsingen, Baldingen St. Georgen (Hartkirch), Bremgarten, Gündlingen, Achkarren, Bickensohl, Schliengen, Welschensteinach.

so Ibid., 334: Vier Kirchsätze Rohrbach, Lotzwil, Ettingen und Egringen; dazu Egerkinden, Aetigen, Waldkirch, Heimiswil, Langenthal, Londiswil und Ursenbach.

51 Ibid., 225: Mußbach I 290 durch Werner Schenk von Ramberg, Wormingen I 304/ I 308 durch die Grafen von Zweibrücken, Kerbach, Bretzingen, Mörlheim, Knerlingen. Zu Heimbach jetzt Martin Armgart, "Die Johanniter von Heimbach: Regionale und überregionale Verbindungen der südpfälzischen Kommende eines Ritterordens während des Mittelalters," Zeitschrift für Geschichte des Oberrheins I 64 (2016): I I I- I 52.

52 Rödel, Das Großpriorat, 272-273, 275: Nidda selbst I I 87, Pfarrkirche Eckensdorf und Kapelle Richardshausen, Pfarrkriche Wallernhausen 1493 durch Landgraf Wilhelm III. von Hessen.

53 Ibid., 28 r: Niederweisel St. Gertrud, Griedel, Ostheim.

s4 Ibid., 289, 29 I: 402 Wildungen, Niederwildungen, dazu Odershausen, Braunau, Reinhardshausen, Kapelle Thilmannshausen.

ss Ibid., 303-304: Sieben Patronatsrechte.

s6 van Winter, Sources, 465: 4 Pfarreien Beverwijk, Heemskerk, Zoeterwoude, Hazerswoude. 
nur noch 40 fratres laici 388 Geistlichen gegenüber, von denen 66 als Weltgeistliche nicht mehr dem Orden angehörten:

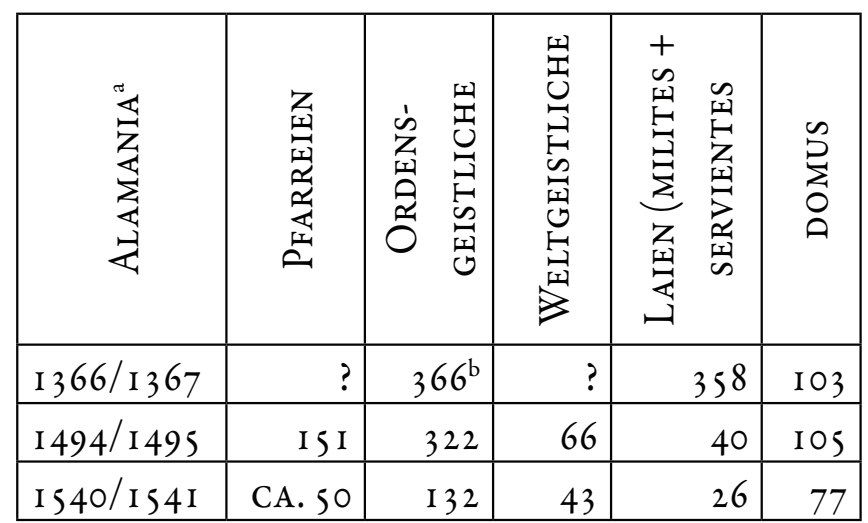

a Borchardt, "Soll-Zahlen," 83-I I 3; Rödel, Das Großpriorat, 4 I o-4I I [ohne Niederlande und Brandenburg].

b Ohne die 68 Schüler, Vikare und Schwestern.

Für die betroffenen Diözesen waren die Johanniter insgesamt bestenfalls von regionaler oder lokaler Bedeutung. Für den Johanniterorden selbst aber sah das, wie die obigen Zahlen belegen, anders aus. Das Priorat Alamania verfügte im späteren Mittelalter über deutlich mehr Pfarrkirchen als Ordenshäuser. Dadurch wurde die im I 4. und I 5. Jahrhundert zunehmende Dominanz des Ritteradels dennoch nicht beeinträchtigt. Herrschend blieben die milites, die von Anfang an de militari genere procreati sein mussten, was sich schrittweise zu formellen Ahnenproben ausweitete, vier, acht, schließlich 16 adelige Ahnen für die Aufnahme als Ritterbruder. ${ }^{57}$ Anders als zum Beispiel in Frankreich spielten fratres servientes in Deutschland praktisch keine Rolle. Ihre Ordensgeistlichen bildeten die Johanniter in größeren Häusern selbst aus, ablesbar an noch nicht zum Priester geweihten Diakonen, Subdiakonen und Schülern, welche in Zeugenlisten von Urkunden oder bei Visitationen genannt werden; die für das Priorat Alamania zusammengestellten Sollzahlen nennen 1367 für Utrecht I o Priester und 6 Schüler, für Sulz im Elsaß 8 Priester und ı o Schüler, I 494/ I 495 für Heimbach bei Speyer I Prior, 6 Priester, I Diakon, I Subdiakon, für Köln 17 Priester, 2 Diakone, I Subdiakon, ${ }^{88}$

57 Hans Karl von Zwehl, Über die Entwicklung der Adelsproben im Souveränen Malteser-Ritterorden: Eine Studie (Essen: Fredebeul \& Koenen, I932).

s8 Dazu Ahn, Die Kölner Johanniterkommende, I I 6- I 26. 
für Freiburg im Breisgau 4 Priester, I Diakon, für Steinfurt in Westfalen 8 Priester und I Diakon. ${ }^{99}$

Ob man diese Konvente - hinzuzufügen wäre das I 37 I eingerichtete Haus zum Grünen Wörth in Straßburg, wo I 495 ein Komtur, ein Prior, I 4 Ordensgeistliche und 18 pauperes femine sive besacce, letztere in dem $\mathrm{I} 38 \mathrm{I}$ durch Konrad von Megede dort gestifteten Spital, lebten ${ }^{60}$ sowie das I 455 errichtete Haus in Biel, wo I 495 neben dem Komtur vier Ordenskapläne lebten ${ }^{61}-$ als zentrale Ausbildungsstätten für Ordensgeistliche ansprechen darf, sei dahingestellt. Im Regelfall dürften nämlich auch kleinere Ordenshäuser, sobald sich Bedarf abzeichnete, einen Priesterkandidaten aufgenommen haben, um ihn nach einiger Zeit auf frei werdende Pfründen zu setzen, welche der Orden zu vergeben hatte. Solche Verhältnisse spiegeln die Soll-Zahlen von I 367 für den Nordosten, in Mirow und Nemerow, Quartschen, Rörchen, Zachan und Schöneck. ${ }^{62}$ Anderwärts mag das ähnlich gewesen sein, nur dass man dort den Weihegrad der Ordensgeistlichen nicht eigens notierte. Hier liegt übrigens auch eine Schwierigkeit, die von Walter Gerd Rödel ausgewerteten, aber nicht edierten Visitationen von I 494/I 495 und I 540/I 54 I zu interpretieren: Die Quelle und ihr Editor reden allgemein von Ordenskaplänen oder Ordensgeistlichen, ohne immer den Weihegrad zu unterschieden.

Wie milites und servientes waren auch die Ordensgeistlichen mit allen drei feierlichen Gelübden, Armut, Keuschheit und Gehorsam, den Ordensoberen verpflichtet. Sie ließen sich leichter kontrollieren als nur in Dienst genommene Weltgeistliche. Und sie mögen auch kostengünstiger gewesen sein. Auf Weltgeistliche jedenfalls griff der Ordens nur aushilfsweise zurück, je länger freilich, desto mehr. Was ein Ordensgeistlicher bekam, wurde selten beziffert. Ein Weltgeistlicher erhielt I 495 vom Komtur in Rheinfelden nur 8 fl, I 54 I dagegen schon i 6 fl. Dabei ist unklar, welche Naturalleistungen daneben anfielen und wie die Unterbringung im Ordenshaus zu veranschlagen war. ${ }^{63}$ Trotzdem: Eine normale Pfründe für einen Weltgeistlichen musste um I 500 rund 50 fl Jahreseinkünfte haben. Man kann sich denken, dass vorwiegend solche Weltgeistliche auf den Johanniterorden hofften, die anderweitig am Pfründenmarkt keine Chancen sahen. Die konkreten Lebensumstände von Ordens- und Weltgeistlichen dürften allerdings gar nicht so unterschiedlich gewesen sein. Beide lebten in den Ordenskonventen, wurden dort versorgt und betreuten von dort aus nahegelegene Kirchen mit oder ohne Seelsorge. Ebenso lebten Ordens- wie Weltgeistliche aufgrund von Bestallung durch

\footnotetext{
59 Borchardt, "Soll-Zahlen," 93-97.

60 Rödel, Das Großpriorat, I 8 I, I 84.

61 Ibid., I07; Helvetia Sacra 4.7.I, I 28 - I 29.

62 Borchardt, "Soll-Zahlen," 95.

63 Rödel, Das Großpriorat, 84.
} 
den Johanniterorden außerhalb von dessen Häusern und Konventen allein oder zu zweit bei weiter entfernt gelegenen Pfarrkirchen, um dort Gottesdienst und Seelsorge zu versehen.

Mindestens sechs Ordenskapläne mussten einen Konvent bilden, damit gemäß den Ordensstatuten dort das Stundengebet abgehalten werden konnte. ${ }^{64}$ Die Anzahl der Patronatsrechte, Inkorporationen und Messpfründen hatte Einfluss auf Anzahl und Stellung der Ordensgeistlichen. Wenn viele Pfarreien und Pfründen oder eine große Stadtpfarrei zu versorgen waren, lebten in einem Ordenshaus naturgemäß mehr Ordensgeistliche, I 494/I 495 in Feldkirch, Leuggern, Buchsee, Biel, Sulz, Duisburg, Arnheim und Nimwegen 4, in Schleusingen 5, in Mergentheim und Sint Jansdal 6, in Sneek 7, im niederrheinischen Wesel, wo die Johanniter von den Franziskanern ein Spital übernommen hatten, in Meisenheim und Utrecht 8, in elsässischen Schlettstadt und Hagenau 8 bzw. 9 und in Heimbach sowie im westfälischen Steinfurt 9, in Straßburg I 6 und in Köln sogar 17 Ordensgeistliche. ${ }^{65}$ Damals zählte man über 300 Kommunikanten in den Ordenspfarreien Altmühlmünster, Mußbach, Kronenburg und Nidda, ${ }^{66}$ wobei allerdings diese Zahl nicht für alle Ordenspfarreien angegeben wurde.

Anders als den servientes kam den clerici immerhin gewisse Bedeutung für die Ordensverwaltung im Priorat Alamania zu. Die meisten und reichsten Ordenshäuser leiteten zwar milites als Komture (preceptores). Damit sie auskömmlich leben konnten, wurden manche früher selbständige Niederlassungen schon im Spätmittelalter als membra einer benachbarten Kommende zugeordnet. So zählte man I 494/I 54 I nur noch 55 Kommenden, I 65024 Kommenden, 178526 Kommenden. ${ }^{67}$ Immerhin 14 Ordensgeistliche, meist Priester, standen I 494/ 4995 als vollgültige Komture einer Kommende vor. Dabei handelte es sich um zehn kleinere Häuser, ( I) Hohenrain mit Johann Reckrode, der sich auf Rhodos befand und durch Raimund von Mainz vertreten wurde, (2) Regensburg mit Johann von Gmünd, der den Ordensgeistlichen Johann Reyner zum Verwalter gemacht hatte, (3) Sobernheim mit Johann Sebenhar, (4) Meisenheim mit Peter Wolfstein, (5) Wiesenfeld und Wildungen mit Johann Rösener, (6) Kronenburg mit Paul Muter,

64 Am 28. August 1495 wurde festgestellt, dass man dies in Steinfurt nicht beachtete: van Winter, Sources, 486.

65 Rödel, Das Großpriorat, 73-74 (Feldkirch), 88 (Leuggern), I04 (Buchsee), I07 (Biel), 20 I (Sulz), 229 (Heimbach), 362 (Duisburg), I 77 (Schleusingen), I6 I (Mergentheim), 368 (Wesel), 25 I (Meisenheim), I 95 (Schlettstadt), 222 (Hagenau), 383 (Steinfurt), I 84 (Straßburg), 3 I 2 (Köln); van Winter, Sources, 405 (Arnheim), 4 I 5 (Nimwegen), 420 (Utrecht), 439 (Sint Jansdal), 457 (Sneek), 475 (Steinfurt).

66 Rödel, Das Großpriorat, I 69, 24 I, 308, 275.

${ }_{67}$ Ibid., 34-35. 
(7) (Nieder)Breisig mit Nikolaus von Trier, der zuvor Prior der Kirche im Kastell St. Peter zu Bodrum an der kleinasiatischen Küste gegenüber von Kos gewesen war, (8) Walsum mit Wichmann Dünstorp, (9) Wesel mit Johann Oesterwick und ( I ) Haarlem mit Johannes Guillerini Johannis. ${ }^{68}$

In Velden bei Düren waltete damals entgegen dem Herkommen ein Ritterbruder als Komtur, Ulrich Vittel, der zugleich Komtur zu Mechelen war ${ }^{69}$; demnach hatte sich bereits im Spätmittelalter eine Tradition herausgebildet, einige Ordenshäuser an fratres clerici als Komture zu übertragen. I 4 Niederlassungen in Friesland, welche von Steinfurt abhingen, durften ihren preceptor selbst wählen, und acht von ihnen wurden I 494/ 1495 von einem Ordensgeistlichen geleitet. ${ }^{70} \mathrm{Hinzu}$ kamen vier größere Niederlassungen, ( I I ) Utrecht, das auf das I 3., vermutlich sogar auf das I 2. Jahrhundert zurückging, ( I 2) Köln, das auf das I 3. Jahrhundert zurückging, wo aber erst seit der Reform von 1469 der Konvent einen Ordenspriester zum Komtur wählen durfte, sowie (I 3 ) Straßburg und (I 4) Biel, die I 37 I und 1455 neu entstanden waren, wo seit der Gründungszeit die Komture durch den Konvent gewählt werden durften. ${ }^{71}$ Offenbar meinten es die Johanniter ernst, wenn sie nach dem Ende der Kreuzzüge im Spätmittelalter auf geistlichem Gebiet tätig wurden. Frühneuzeitlich verfestigten sich sogenannte Priesterkommenden, die regelmäßig an fratres clerici fielen. Nach Christian von Osterhausen, Eigentlicher und gründlicher Bericht (1650) waren das neun ausreichend dotierte Häuser oder Doppelhäuser, und zwar in Alamania superior (I) Straßburg mit Schlettstadt, (2) Regensburg mit Altmühlmünster, (3) Worms, (4) Schleusingen mit Weißensee, (5) Freiburg im Üchtland, (6) Sobernheim mit (Hangen)Weisheim, in Alamania inferior (7) Köln, (8) Aachen und (9) Roth mit Kronenburg. ${ }^{2}$ Hätten die Johanniter nämlich ihren Priestern Aufstiegschancen grundsätzlich verwehrt,

68 Ibid., 96, 165, 248, 25 1, 287, 290, 307, 345, 365, 367; van Winter, Sources, 465 (Haarlem).

69 Rödel, Das Großpriorat, 335 .

70 van Winter, Sources, 476-479 für Jemgum, Hasselt, Boekzetel, Burmönken, Hove, Muhde, Bredehorn, Bokelesch, Langholt.

${ }_{71}$ In Köln Hubert von Heinsberg I 469-I 496, Konrad Bucker I 499-I 5 I 2, Peter von Simmern I 5 I 3-I 52 I, Arnold von Kronenburg I 522-I 540, in Straßburg Nikolaus von Baden I 495 und I 540 Georg Beidt, in Biel 1494 Stephan Lang: Rödel, Das Großpriorat, I07, I 84-i 85 , 3 I I; Ahn, Die Kölner Johanniterkommende, I I 9- I 20; Helvetia Sacra 4.7. I, I 28 und I 53 . In Utrecht Fr. Alexander de Rey, Ordenskaplan, Komtur und zugleich Bailli von Utrecht: van Winter, Sources, 419-420.

72 Christian von Osterhausen, Eigentlicher und gründlicher Bericht dessen was zu einer volkommenen Erkantnuß deß hochlöblichen Ritterlichen Ordens S. Johannis von Jerusalem zu Malta vonnöthen (Augsburg: Andreas Aperger, 1650), 790-794. Im Ruolo dei Cavalieri von 1785 (Malta, AOM 2200) werden nur noch fünf Priesterkommenden genannt: Aachen, Regensburg, Freiburg im Üchtland, Roth bei Vianden und Worms. Rödel, Das Großpriorat, 34-35 mit Anm. I30-I 3 I. 
wäre ihnen zweifellos der infolge der Reformation ohnehin zurückgehende Priesternachwuchs vollends weggebrochen.

Priesterbrüder als preceptores waren aber nicht alles. Während des Spätmittelalters fanden Ordensgeistliche ausweislich der Visitation I 494/ I 495 außerdem Verwendung als Verwalter von membra, ${ }^{73}$ kleinen Niederlassungen, welche zu arm waren, um einen Komtur standesgemäß unterhalten zu können. Schleusingen verwaltete damals der Ordensgeistliche Johann Beyer, weil der Komtur Johann Wilhelm von Bodman zugleich die Kommende Kleinerdlingen besaß und dort residierte. ${ }^{74}$ In Hagenau und in Nidda nahm der Prior des dortigen Priesterkonvents zugleich die Aufgaben eines Verwalters wahr. ${ }^{75}$ In Rexingen, Bassel, Grebenau und Horst an der Lippe übten jedoch Ordensbrüder, welche keine clerici waren, ${ }^{76}$ in Biberstein und in Heitersheim sogar weltliche Schaffner das Verwalteramt aus. ${ }^{77}$ $\mathrm{Ab}$ der Reformationszeit wurden weltliche Verwalter aufgrund des um sich greifenden Priestermangels zur Regel; solange der Orden jedoch über genügend fratres clerici verfügte, waren die Ordensgeistlichen eine Alternative zu den später allein dominierenden weltlichen Schaffnern oder Pächtern (arrendatores).

73 Rödel, Das Großpriorat, 66 (Wädenswil), 73 (Feldkirch), 84 (Rheinfelden), 99 (Reiden), I 37 (Dätzingen), I 69 (Altmühlmünster, ernannt auf einem Provinzialkapitel in Frankfurt durch Fr. Johann von Cardona, Bailli von Mallorca), i 95 (Schlettstadt), 206 (Mülhausen), 235 (Weißenburg), 238 (Bruchsal), 240 (Mußbach), 254 (Mußbach, ernannt auf Lebenszeit durch den Vorgänger des jetzigen Komturs zu Meisenheim ohne Zustimmung des Priors und des Provinzialkapitels), 255 (Tiefenbach), 26I (Hangenweisheim), 267 (Mosbach, ernannt auf Lebenszeit durch den Komtur von Frankfurt), 293 (Pfannstiel), 300 (Roth bei Vianden), 329 (Neuenburg), 33 I (Kenzingen), 334 (Thunstetten), 340 (Nideggen), 342 (Kieringen), 347 (Hönningen), 352 (Aachen, der einzige dort lebende Ordensbruder Nikolaus Kochemß, Prior von Adenau), 358 (Herrenstrunden, der Ordenskaplan Gottschalk Vese, ernannt auf Befehl des Großmeisters am I9. Juli I 480 in Köln durch Fr. Johann von Cardona, Bailli von Mallorca), 359 (Marienhagen), 390 (Münster); van Winter, Sources, 400-40 I, 403-404 (Aachen), 405 (Arnheim), 439 (Sint Jansdal), 445 (Oudewater), 447 (Waarder), 449 (Harmelen), 450 (Wemeldinge), 453 (Kerkwerve, ein Nonnenkonvent), 455 (Middelburg), 457 (Sneek).

74 Rödel, Das Großpriorat, I77, I 49.

75 Ibid., 222, 274.

76 Ibid., I 3 I (Konrad von Wonneck in Rexingen), 2 I 8/20 (Johann Wyck in Bassel, bestellt I 486 durch den Komtur von Dorlisheim mit Zustimmung durch Prior und Provinzialkapitel), 279 (Gerlach von Wahlen in Grebenau). Hier ist unklar, ob es sich jeweils um einen frater miles oder einen frater serviens handelte, ibid., 39 I (Horst an der Lippe, der miles Fr. Heinrich Drost).

77 Ibid., 90, 325 . 


\section{SCHLUSSBEMERKUNG}

Als im I 6. Jahrhundert durch die Reformation die Zahl altgläubiger Priesteraspiranten drastisch einbrach, entstand für die Johanniter und ihre Pfarreien im Priorat Alamania eine neue Situation. Hinzuweisen ist hier vor allem auf das Bemühen neugläubiger Obrigkeiten, die Johanniter von der Seelsorge in ihren Territorien auszuschließen. ${ }^{7}$ Darin lag ein wesentliches Motiv für protestantische Territorien und Reichsstädte, die Johanniter zu enteignen. Gerade deshalb verlieh Kaiser Karl V. I 548, auf dem "geharnischten Reichstag" in Augsburg, dem Großprior Georg Schilling von Cannstatt formell den Rang eines Reichsfürsten. So wurde die Beschlagnahmung von Ordensbesitz deutlich erschwert. Mitunter aber zwang man den katholischen Orden, lutherische Geistliche zu besolden, so die Herzöge von Kleve in Duisburg, die Landgrafen von Hessen in Nidda oder die Reichsstadt Rothenburg in Reichardsroth. ${ }^{9}$ Grundbegriffe und Grundstrukturen, wie sie sich im hohen und späteren Mittelalter ausgebildet hatten, wirkten mithin in der Frühneuzeit fort bis zur Säkularisation an der Wende vom i 8. zum i 9. Jahrhundert.

\section{QUELLENANHANG}

Reichardsroth, Rothenburg/Rothenburg, 7/I8. Mai 1464.

Instrument des Notars Johann Neumann, Klerikers der Diözese Mainz, über Aussagen, welche er auf Bitten des Fr. Michael Lotter, Priors und Prokurators des Fr. Georg von Melchingen, Komturs der Häuser Rothenburg und Reichardsroth, schriftlich festgehalten hat. Ausgesagt haben (I) Fr. Adam Kobolt, Johanniterkonventuale in

78 Karl Borchardt, "Die Johanniter in Deutschland und die Reformation," in The Military Orders and the Reformation: Choices, State Building, and the Weight of Tradition, Papers of the Utrecht Conference, 30 September - 2 October 2004, hrsg. v. Johannes A. Mol, Klaus Militzer und Helen J. Nicholson, Bijdragen tot de Geschiedenis van de Ridderlijke Duitsche Orde, Balije van Utrecht 3 (Hilversum: Verloren, 2006), I I I- I 7 .

79 Nordrheinisches Klosterbuch, 2: I6 I (Duisburg); Rödel, Das Großpriorat, 273-274 (Nidda); Karl Borchardt, "Reichardsroth zur Zeit der Johanniter," in Kirchen, Bürger und Bauern: Ausgewählte Studien zu Rothenburg ob der Tauber und seinem Umland, hrsg. v. Karl Borchardt, Jahrbuch des Vereins Alt-Rothenburg (Rothenburg: Verein Alt-Rothenburg, 20 I6), I 24-I 39 , hier I 36, zuerst erschienen: Reichardsroth und sein Johannitererbe 1254-2004, hrsg. v. Ev.-Luth. Pfarramt Langensteinach (Uffenheim: Ev.-Luth. Pfarramt Langensteinach, 2004), S. 54-65 (Reichardsroth). 
Rothenburg, und Konrad Kühlwein, Laie aus der Diözese Würzburg, (2) Fr. Engelhard Hertrich und Fr. Georg Trittrauf, zwei Johanniterkonventualen in Rothenburg. Sie erklärten übereinstimmend, Fr. Friedrich Fetzer von Geispitzheim, Komtur zu Mergentheim und Generalrezeptor der Ballei Franken, habe von dem verstorbenen Fr. Georg von Scharenstetten, der über zo Jahre bis zu seinem Tode Statthalter des Hauses Reichardsroth war, genannte Fahrnis in Crailsheim, 700 fl ausstehende Schulden sowie 6oo Malter Getreidevorräte in den Kornhäusern zu Windsheim und Rothenburg eingenommen. Die beiden letzteren Konventualen erklärten zudem, Fr. Georg von Scharenstetten habe ein Viertel des Zehnten und weitere Gefälle in Ellrichshausen gekauft und damit zwei Pfründen in Crailsheim gestiftet, welche künftig der Komtur von Rothenburg zu verleihen habe. Zeugen waren am 7. Mai zur ersten Stunde in Reichardsroth Ulrich Strebel und Konrad Schmidt, in Rothenburg zur Vesperzeit Heinrich Ortlin der Ältere, Heinrich Ortlin der Jüngere und Johann Marstaller, am I8. Mai zu Rothenburg in der Pfarrkirche Johann Mangolt, Altarist im Frauenkloster zu Rothenburg, und Nikolaus Müller, Pfarrer in (Tauber)Schekkenbach in der Diözese Würzburg.

Ausfertigung: BayHStAM RiU 242.

Größe: $35 \mathrm{~cm}$ breit, 58,4 cm hoch; Pergament; beglaubigt durch das Signet des Notars.

In nomine Domini amen. Anno a nativitate eiusdem millesimo quadringentesimo sexagesimo quarto, indictione duodecima, die vero Lune septima mensis Maii, hora primarum vel quasi, pontificatus sanctissimi in Christo patris et domini nostri domini Pii divina providentia pape secundi anno eius sexto in mei notarii publici testiumque infrascriptorum ad hoc specialiter vocatorum et rogatorum presentia personaliter constitutus religiosus dominus Michael Lotter prior et procurator domus sancti Iohannis Ierosolimitani opidi Rotenburg an der Tauwer Herbipolensis diocesis de mandato et ex commissione religiosi fratris Georii de Melchingen commendatoris iamdicti domus et domus in Reichartzrod ordinis sancti Iohannis predicti et nonnullos testes de et super punctis et articulis infrascriptis, videlicet dominum Adam Cobolt conventualem dicti domus in Rotenburg et Conradum Külwein laycum Herbipolensis diocesis predicte coram me notario et testibus infrascriptis produxit et eosdem testes examinari ac dicta et deposiciones eorum in publicam formam redegi et exemplari debita cum instantia petiit ac postulavit. Tenor articulorum sequitur et est talis:

Item quod veritas fuit et est preter id et absque eo, quod frater Fridericus Fetzer de Geyswitzen conmendator domus in Mergethein ordinis sancti Iohannis Ierosolimitani predicti dixit et reputavit se esse generalem receptorem sacri con- 
ventus Rodi per palegiam Franckonie. Item quod idem frater Fridericus omnia et singula bona mobilia et immobilia, lectisternia, pulvinaria, cussinas, linteamina, tecturas lectisterniorum, canistrum scutellas, flasculas necnon omnia alia et singula utensilia domus per quondam dominum Georium de Scharensteten locumtenentem eo vivente domus in Reichartzrod predicte diocesis in opido Creuelßhein post mortem ipsius Georii relicta et dimissa. Item etiam quod dictus frater Fridericus Feczer recepit et sublevavit a debitoribus ipsius fratris Georii septingentos florenos Renenes, quod ipse Georius post mortem reliquit et dimisit. Item dictus frater Fridericus recepit sexingenta maldra singulorum fructuum vel circa iacentia in granariis opidorum Winßhein et Rotenburg, que frumenta ipse frater Georius post mortem suam reliquit. Item ultra premissa veritas fuit et est, quod sepenominatus frater Georius de Scharensteten ut locumtenens prefati domus in Reichartzrod fuit in pacifica et quieta possessione prefati domus plus quam ad viginti annos et usque ad tempus obitus sui fructusque, redditus et proventus prefati domus in Reichartzrod percepit et sublevavit illosque in usus suos convertit sine contradictione cuiuscumque etcetera.

Quibus testibus sic coram me notario infrascripto per prefatum dominum Michaelem priorem productis et exhibitis ipse dominus prior prefato domino Adam conventuali sub pena obedientie, ut quidquid sibi de supradictis punctis et articulis constaret, non odio vel favore pretermittendo, quod factum veritati impedire posset, mandavit atque eundem conpulit. Huiusmodi namque conpulsione sic ut premittitur facta ipse dominus Adam deposuit ut sequitur:

Et primo reducto ipsi domino Adam iuramentum credientie ad memoriam et causam stare sui dicti allegans dixit verum esse, quod frater Fridericus Feczer conmendator domus in Mergethein dixisset se esse generalem receptorem sacri conventus Rodi per palegiam Franckonie seque pro tali habuit, tenuit et nominavit et ideo dixit se habere in mandatis recipiendi et sublevandi omnia et singula bona mobilia et inmobilia, frumenta et etiam debita per quondam fratrem Georium de Scharensteten supranominatum relicta et dimissa. Item ipse dominus Adam dixit esse verum, quod dictus frater Fridericus Feczer conmendator recepisset et sublevasset septingentos florenos auri Renenses vel circa, quos prefatus frater Georius de Scharensteten post mortem suam in debitis reliquit. Item dixit esse verum ipse dominus Adam, quod predictus frater Fridericus recepisset et sublevasset sexingenta maldra diversorum frumentorum vel circa per ipsum fratrem Georium in granariis opidorum Winßhein et Rotenburg relicta et dimissa ${ }^{a}$. Item dixit esse verum, quod antenominatus frater Fridericus omnia et singula bona mobilia et inmobilia eciam utensilia domus, videlicet lectisternia, pulvinaria, linteamina, tec-

a dimissis irrig im Text 
turas lectisterniorum, canistrum scutellas, $\mathrm{v}[1]$ asculas, quorum omnium numerum et valorem se scire non dixit, eciam argenteria, que plus quam centum florenos auri Renenes valoris fuissent, recepisset et in usos suos convertisset. Item ultimo dixit esse verum ipse dominus Adam, quod frater Georius de Scharensteten ut locumtenens in pacifica et quieta possessione antedicti domus in Reichartzrod quasi ad viginti annos et usque ad tempus obitus sui fructusque, redditus et proventus ex eadem domo in Reichartzrod provenientes recepisset, sublevasset et in usos suos convertisset sine contradictione ordinis aut alterius cuiuslibet etcetera.

Deinde adstatim eadem hora et die ut supra Conradus Kulwein supradictus laycus Herbipolensis diocesis in $\mathrm{XL}^{\mathrm{mo}}$ vel circa sue etatis anno constitutus medio suo iuramento per eum tunc ad mandatum et in manibus mei notarii prestito deposuit super suprascriptis punctis et articulis ut sequitur: Et primo causam scientie sui dicti allegans dixit verum esse, quod frater Fridericus Feczer conmendator domus in Mergethein dixisset se esse generalem receptorem sacri conventus Rodi per palegiam Franckonie seque etiam pro tali habuit, tenuit et nominavit et ideo dixit se habere in mandatis et potestate recipiendi et sublevandi omnia et singula bona mobilia et inmobilia et debita quecunque per quondam dictum Georium de Scharensteten post mortem relicta et dimissa ${ }^{b}$. Item ipse Conradus Kulwein dixit esse verum, quod frater Fridericus Feczer recepisset et sublevasset septingentos florenos auri Renenses, quos prefatus frater Georius in debitis post mortem reliquit et dimisit. Item ipse Conradus dixit esse verum, quod antedictus frater Fridericus sexingenta maldra diversorum frumentorum vel circa iacentia in granariis opidorum Rotenburg et Winßhein recepisset et sublevasset. Item dixit esse verum, quod frater Fridericus Feczer omnia et singula bona mobilia et inmobilia eciam omnia utensilia domus necnon argenteria valoris plus quam centum florenos auri Renenses per ipsum fratrem Georium in opido Kreuelßhein Herbipolensis diocesis, ubi dictus frater Georius diem vite sue clausit extremum, recepisset et sublevasset et sibi illa inbursasset. Item ipse Conradus Kulwein dixit esse verum, quod frater Georius de Scharensteten ut locumtenens in pacifica et quieta possessione dicte domus in Reichartzrod ad viginti annos vel circa et usque ad tempus obitus sui fuisset seque pro talem habuisset, tenuisset et nominasset fructusque, redditus et proventus ex eadem domo in Reichartzrod provenientes recepisset et sublevasset et in usos suos convertisset sine contradictione cuiuscunque etcetera.

Acta sunt hec in stuba curie domus habitacionis domus in Reichartzrod sub anno ut supra presentibus ibidem providis viris Vlrico Strebel et Conrado Smit laycis Herbipolensis diocesis predicte testibus ad hoc vocatis et ${ }^{\mathrm{c}}$ rogatis.

b dimissis irrig im Text

c et irrig verdoppelt 
Deinde anno, indictione, die, mense et pontificatu quibus supra hora vesprorum vel quasi in stuba maiori curie domus sancti Iohannis Ierosolimitani opidi Rotenburg an der Tawber antedicte Herbipolensis diocesis constitutus prefatus dominus Michael prior et procurator iamdicti domus et nonnullos alios testes, videlicet dominos Engelhardum Hrettrich ${ }^{\mathrm{d}}$ et Georium Trittrauff conventuales dicti domus sancti Iohannis in Rotenburg, coram me notario infrascripto et testibus infrascriptis produxit illosque per me notarium de et super omnibus et singulis punctis et articulis suprascriptis examinari petiit et postulavit mandans nichilominus dictus dominus Michael prior prefatis dominis Engelhardo et Georio sub pena obedientie prestite, ut quidquid ipsis de supradictis punctis et articulis constaret, non odio vel favore pretermittendo, quod factum veritati impedire posset, veritatem dicerint et deponerent.

Huiusmodi conpulsione $e^{\mathrm{e}}$ sic ut premittitur per prefatum dominum Michaelem facta dominus Engelhardus Herttrich conventualis dicti domus in Rotenburg reducto sibi prius obedientiam ad memoriam deposuit ut sequitur: Primo causam scientie sui dicti allegans dixit verum esse, quod frater Fridericus Feczer dixisset se esse generalem receptorem sacri conventus Rodi per palegiam Franckonie et ideo habere in mandatis recipiendi et sublevandi omnia et singula bona mobilia et inmobilia eciam debita et frumenta per quondam fratrem Georium de Scharensteten relicta et dimissa. Item idem dominus Engelhardus dixit esse verum, quod antedictus frater Fridericus Feczer septingentos florenos vel circa, quos dictus frater Georius post mortem suam in debitis reliquit et dimisit, recepisset et sublevasset. Item dixit eciam ipse dominus Engelhardus esse verum, quod frater Fridericus Feczer sexingenta maldra diversorum frumentorum vel circa iacentia in granariis opidorum Rotenburg et Winßhein, que eciam ipse Georius post mortem reliquit. Item dixit etiam esse verum, quod frater Georius de Scharensteten dicte domus in Reichatzrod plus quam ad viginti annos vel circa et usque ad tempus obitus sui fructusque, redditus et proventus ex predicta domo provenientes sine impedimento et contradictione dicti ordinis recepisset, sublevasset et imbursasset et in usos suos convertisset. Item ultra premissa dictus dominus Engelhardus dixit esse verum, quod sepenominatus frater Fridericus Feczer de omnibus bonis et utensilibus domus per ipsum quondam Georium de Scharensteten relictis et dimissis non plus dedit et reliquit domui sancti Iohannis in Rotenburg et conventualibus eiusdem domus quam tria lectisternia et sex linteamina et unum almare, in quo ponuntur ornamenta ecclesie.

d huiusmodi conpulsione irrig verdoppelt und dann expungiert

e Herttrich richtig 
Deinde adstatim dominus Georius Trittrauff reducto sibi prius iuramentum obedientie ad memoriam deposuit ut sequitur: Primo causam scientie sui dicti allegans dixit esse verum, quod frater Fridericus Feczer dixisset se esse generalem receptorem sacri conventus Rodi et ideo se habere in mandatis percipiendi et sublevandi omnia et singula bona mobilia et inmobilia, etiam debita et frumenta per quondam fratrem Georium de Scharensteten relicta et dimissa post mortem suam. Item dixit etiam esse verum, quod ipse frater Fridericus septingentos florenos Renenses, quos antedictus frater Georius in debitis reliquit post mortem suam, recepisset et sublevasset. Item dixit etiam esse verum, quod ipse frater Fridericus $\mathrm{VI}^{\mathrm{c}}$ maldra diversorum frumentorum vel circa iacentia protunc in granariis opidorum Rotenburg et Winßhein per ipsum fratrem Georium post mortem suam etiam relicta ${ }^{f}$ et dimissa recepisset et sublevasset. Item ipse dominus Georius dixit esse verum, quod sepenominatus frater Georius in pacifica et quieta possessione prefati domus in Reichartzrod ad viginti annos vel circa et usque ad tempus obitus sui fuisset fructusque, redditus et proventus ex eadem domo provenientes sine contradictione dicti ordinis semper percepisset et sublevasset. Item ultra premissa dixit esse verum, quod prefatus frater Fridericus omnia et singula bona mobilia et inmobilia necnon utensilia domus et presertim argenteria, que plus quam centum florenos auri valoris fuerunt, quos dictus frater Georius in opido Kreuelßhein, ubi ipse Georius diem vite sue clausit extremum, reliquit et dimisit, recepisset et sublevasset et de omnibus bonis et utensilibus domus ipse frater Fridericus solum reliquit et dedit domui in Rotenburg et conventualibus eiusdem tria lectisternia, sex linteamina et unum almare, in quo ponuntur ornamenta ecclesie dicti domus, et non plus. Presentibus ibidem providis viris Henrico Ortlin seniori et iuniori et Iohanni Marstaller laycis dicte Herbipolensis diocesis testibus vocatis et rogatis.

Postremo vero anno, indictione, mense et pontificatu quibus supra, die vero Veneris, que fuit decimaoctava mensis supradicti, in ecclesia parrochiali antedicti opidi Rotenburg memoratus dominus Michael prior et iamdictos dominos Engelhardum Herttrich et Georium Trittrauff conventuales de et super emptione quarte parte decime et nonnullis aliis censibus et redditibus in villa et marchia Erlingßhausen Herbipolensis diocesis pro fundatione duorum beneficiorum per prefatum fratrem Georium de Scharensteten emptis et conparatis et post mortem dicti Georii collationem dictorum beneficiorum ad commendatorem domus in Rotenburg spectantium et pertinentium per me notarium infrascriptum examinari petiit et postulavit, mandans ipse dominus Michael prior iamdictus testibus sub pena obedientie, ut, quidquid ipsis de premissis constaret, veritatem dicerent, conpulit.

f relictis irrigim Text 
Huiusmodi conpulsione sic facta Engelhardus deposuit ut sequitur: Item dixit sibi bene constare et esse verum, quod sepenominatus dominus Georius de Scharensteten emisset quartam partem decime cum nonnullis censibus et redditibus in villa et marchia Erlinghausen pro fundatione duorum beneficiorum in opido Kreuelßhein et quod collatio ipsorum post mortem ipsius fratris Georii deberet spectare et pertinere ad conmendatorem dicti domus in Rotenburg.

Item adstatim dominus Georius Trittrauff deposuit super premissis ut sequitur: Item dixit sibi bene constare et verum esse, quod frater Georius de Scharensteten emisset quartam partem decime cum nonnullis aliis censibus et redditibus in villa et marchia Erlingßhausen pro fundatione duorum beneficiorum in opido Kreuelßhein et quod collatio ipsorum post mortem ipsius fratris Georii deberet et debet spectare et pertinere ad conmendatorem pro tempore existentem dicti domus sancti Iohannis opidi Rotenburg etcetera.

Super quibus omnibus et singulis memoratus dominus Michael prior et procurator assertus prefati fratris Georii de Melchingen conmendatoris pretacti domus in Rotenburg sibi super premissis unum vel plura publicum seu publica fieri petiit instrumentum et instrumenta. Acta sunt hec anno, indictione, die, mense et pontificatu quibus supra, hora meridiei vel quasi presentibus ibidem honorabilibus viris dominis Iohanni Mangolt altarista monasterii beate Virginis monialium opidi Rotenburg sepedicti et Nicolao Molitoris plebano in Scheckenbach Herbipolensis diocesis antedicti testibus ad premissa vocatis pariter et rogatis.

[Signet] Et ego Iohannes Newman clericus Maguntinensis diocesis publicus sacra imperiali auctoritate notarius, quia predictorum testium productioni, receptioni, conpulsioni et examinationi omnibusque aliis et singulis premissis, dum sic ut premittitur fierent et agerentur, una cum $^{\mathrm{g}}$ prenominatis testibus presens interfui eaque omnia et singula sic fieri vidi, audivi et per me ipsum feci, idcirco hoc presens publicum instrumentum manu alterius me aliis occupatis negociis fideliter scriptum exinde confeci, subscripsi, publicavi et in hanc publicam formam redegi signoque et nomine meis solitis et consuetis signavi in fidem et testimonium omnium et singulorum premissorum rogatus et requisitus.

Basel, 6. Juni 1464.

Fr. Johann von Ow, Statthalter des Meisters Fr. [Richard von Buttlar], und die Pfleger aufihrem Kapitel in Basel erklären, Fr. Georg von Melchingen, Komtur zu Rothen-

g pre irrig 
burg, habe vor ihnen gegen Fr. Hermann von Hunwil, Komtur zu Hall, als bevollmächtigten Prokurator des Fr. Johann von Sachsenheim geklagt, weil der verstorbene Fr. Georg von Scharenstetten ein Viertel des Zehnten in Ellrichshausen und anderes für die Stiftung zweier Pfründen in Crailsheim gekauft habe, welche das Haus Rothenburg zu verleihen habe. Fr. Hermann von Hunwil antwortete darauf, Fr. Georg von Scharenstetten habe nur neun Jahre die Kommende Rothenburg verwaltet und sie nach Ablauf dieser Frist allein deshalb weiter besessen, weil man ihm Geld schuldig war; er sei aber immer Konventsbruder in Erdlingen gewesen, weshalb die beiden Pfründen durch das Haus Erdlingen zu verleihen seien. Fr. Georg von Melchingen verwies dagegen auf Urkunden, nach denen beide Pfründen mit Gütern des Hauses Rothenburg gestiftet worden seien. Auf Wunsch beider Seiten wurde der Komtur von Robrdorf als Zeuge gehört. Am Ende entschieden die Aussteller, die Verleibung der beiden Pfründen stehe dem Hause Rothenburgzu. Siegel des Priorats [Deutschland]. Ausfertigung: BayHStAM RiU 243.

Größe: $38,3 \mathrm{~cm}$ breit, 21,2 cm hoch, dazu 2,9 cm Plica; Pergament; ein Siegel, anhängend, erhalten, beschädigt, rund, $52 \mathrm{~mm}$, im Sechspass ein Ritter kniend vor Johannes dem Täufer, zu Füßen des Ritters Wappenschild Ow, Umschrift gotische Minuskel:

†. S(igillum) ... prioris . et . prioratus . almanie . ordinis . s(ancti) . ioh(an)nis

Wir brůder Johans von Ouw, statthalter des meisters, und gemeine pfleger und stattåltere in tůtschen landen sant Johans ordens des heiligen spitals von Jerusalem tůnd kund alle[n], so disen brieff ansehent, lesent oder hơrent lesen, das fur uns in unser gesessen cappittel komen sind die ersamen geistlichen brůder Jerg von Melchingen, comenthůr zů Rottenburg uff der Thuber, uff ein und bruder Herman von Hůnwil, comendůr zů Hall egeschribens ordens, als in namen und an statt ein volmechtiger procurator des ersamen geistlichen brůder Johans von Sachsenheim, zů der anderen sitten. Und clagte do der genant brůder Jerg, comenthůr zů Rotenburg, durch sinen fursprechen, wie das vor ettlichen durch den ersamen geistlichen bruder Jergen von Schorenstetten ord(ens) vorgeschribens seliger gedechtniß ettlich rent und gült erkoufft sint, namlich ein quart des zehenden in dem dorff und marckt Erlingeßhusen mit ettlichen anderen zinsen, zů einer fundacion zweyer pfrunden in dem flecken Kråwelßhein, welchen pfrůnden dem huß zů Rotenburg zůstandent ze lyhen, als denn solichs von dem obgenanten bruder Jergen Schornstetten geordeniert worden sy. Und zougt ouch domit ein instrument, darinne begriffen ist ein kuntschafft wisend, das die lihung und collation zů dem vorgenanten huse Rotenburg gehơren sỏll mit mer worten. Daruff der obgenante brůder Herman von Hůnnwil als ein procurator und in procurators wise 
und namen des genanten brůder Johansen von Sachsenheim antwort in sólch meinung, wie das die commenthůrey zů Rotenburg von ettlichen zitten brůder Jergen von Schornstetten nůn jor lang gelihen sy, also wenn die nůn jor verschinen und vß syent, das er denn mit sollcher commenthury nutzit mer ze schicken haben sóll, das derselb brůder Jerg selb erkant hab in cappittel und anderen enden, und das er ein couentbrůder zů Erlingen sy. Nůn nach den nůn joren hat noch denn der Schornstetter sólich huß ingehalten, aber allein umb ein namlich sum, die man im doby schuldig were, wenn im die weren, welle er geren dovon ston. Indem sy der genant brůder Jơrg als von tod abgangen. Deshalb er ein couentbrůder zů Erlingen gewesen und die obgedachten pfrůnden zů Kråwelßhein zelihen gen Erlingen gehơrent, und nit gen Rotenburg. Dowider der comenthůr von Rotenburg aber ließ reden zů glicher wiß als uor und souil me, brůder Jórg von Scho̊rnstetten hab solich zinß erkoufft von dem gůt, das er von der comenthůry Rotenburg gewunnen hab, dodurch und nach lut und sag des instrumentz dasselb gůt und pfrůnden gon Rotenburg gehorent. Und begerten do bedteil darumb den comenthůr von Rordorff ${ }^{\mathrm{h}}$ ze hơren. Wartt ouch darumb gehơrt. Dowider der egemelt comenthůr von Hall in namen und an statt brůder Johanns von Sachsenheim ret in aller der wiß und maß wie vor, das der genant brůder Jerg von Schornstetten ein couentbrůder zů Erlingen gewesen; wer deßhalb solich sin verlassen gůt dem huß Erlingen zůgehore und nit dem huß Rotenburg mit me wortten, nit all not ze schriben. Und wir sy zů beder sitten gnugsamcklich verho̊rt und sy das zům rechten gesetzt hand. Und nach verhorung instrument und ander kuntschafft haben wir darumb mit einhelliger urteil erkent und sprechent zů recht, das die lehenschafft der pfrunden zů Kråwelheim, an der brůder Jo̊rg von Schornstetten seliger gedechtniß die quart des zehenden und ander gúlt oder zinß in dem dorff Erlingßhusen und an anderen enden erkoufft hot, an unsers ordens huß zů Rotenburg uff der Thuber gehơren solle und nit an das huß Erlingen. Des zů warem urkůnd haben wir unsers gemeinen prioratz insigel gehenckt an disen brieff, der geben ist in unserem cappittel zů Basel gehaltten vuff fritag nach unser Frouwen tag Visitationis nach der geburt Cristi vierzehenhundert sechtzig und vier jare gezalt etcetera.

h Fr. Georg Bombast von Hohenheim I 453-1 496: Paul Friedrich Stälin, "Komture des Johanniter-Ordens im Gebiet des jetzigen Königreichs Württemberg," Archivalische Zeitschrift I/8 (I883): I I 0 . 
Basel, 23. August I466.

Fr. Johann von Ow, Großbailli des Konvents zu Rhodos und Statthalter des Fr. Richard von Buttlar, Meisters in deutschen Landen, sowie die Pfleger auf dem Kapitel zu Speyer erklären, Fr. Hermann von Hunwil, Bailli zu Franken und Komtur zu Hall, habe nach Ordensgewohnheit das Recht verlangt, die durch Fr. [Georg von] Scharenstetten gestiftete Pfründe in Crailsheim verliehen zu dürfen. Dagegen habe Fr. Georg von Melchingen, Komtur zu Rothenburg, auf das zu Basel ergangene Urteil verwiesen, wonach die Verleihung der Pfründen dem Komtur von Rothenburg zustehe. Da Urteile einander nicht widersprechen dürfen, wird die frühere Entscheidung bestätigt. Siegel des Priorats [Deutschland].

Ausfertigung: BayHStAM RiU 244.

Größe: 21,2 cm breit, 26,7 cm hoch, dazu $4 \mathrm{~cm}$ Plica; südländisches Pergament; ein Siegel anhängend, stark beschädigt (wie oben Nr. 2).

Wir Johans von Awe, groß balye des heiligen couents zu Rhodis, statthalter des hochwirdigen herrn herrn Richart von Bütlar sant Johans ordens meister in tutzschen landen, und die gemein pfleger im capitel zu Spier gehalten bekenn und thun kund offentlich mit disem brieff gein allermeniglichen, als bruder Herman von Hunwil, balyer in Francken und comenttur zu Halle, fur uns komen und anbracht von wegen einer pfrund zu Kreulshein gelegen, die der Scharenstetter gestifft hat selig ingetrawen, das solich pfrund ime und sinem huse nach ordens recht und gewonheit zugeuallen und von demselben seinem hawse die zuuerleyhen zu lehen gen sollen und begert darumb gerichts. Das veranttwurt bruder Jorg von Melchingen, comenthur zu Rotenburg, wie er solich gemelt pfrund vormals im capittel zu Basel mit recht erlangt und erwonnen. Invortruwen kein urteil wider das ander geen, sunder der genante comenthur umb den zuspruch nicht schuldig und das gerurt urteil in dem gemelten capitel zu Basel ergangen billich bekreffiget werden solle, haben wir nach clag, antwort, rede, wider rede und allem handel zu recht erkant, das das vorgesprochen urteil im capitel zu Basel ergangen bey krefften und wirden bleiben und die gemelten pfrund in Kreulsheim von dem hawse und comenthuri zu Rotenburg ze lehen geen sollen. Des zu warem urkund haben wir unser gemein priorat insigel an disen brieff loussen thün hencken, der geben ist am samstag nachst vor Bartholomei, als man zalt nach Cristi unsers lieben herrn geburt tusent vierhundert und darnach in dem sachs und sechtzigsten jaren. 


\section{Primary SOURCES:}

München. Bayerisches Hauptstaatsarchiv, Ritterorden Urkunden 238, 242, 243, 244, 253. Würzburg. Diözesanarchiv, S [= Schöffel, Abschriften] I.

Beschreibung des Oberamts Crailsheim. Herausgegeben von K. statistisch-topographisches Bureau. Stuttgart: Kohlhammer, 1884.

Brandenburgisches Klosterbuch: Handbuch der Klöster, Stifte und Kommenden bis zur Mitte des I6. Jabrhunderts. Herausgegeben von Heinz-Dieter Heimann, Klaus Neitmann, und Winfried Schich. Brandenburgische Historische Studien I 4. Berlin: be.bra wissenschaft, 2007.

Cartulaire général de l'Ordre des Hospitaliers de S. Jean de Jérusalem. 4 Bde. Herausgegeben von Joseph Delaville le Roulx. Paris: Leroux, I 894/ 1906.

Corpus Iuris Canonici. 2 Bde. Herausgegeben von Emil Friedberg. Leipzig: Tauchnitz, I 879/I 88 I.

Hanauer, Charles Auguste. Cartulaire de l'Église S. George de Haguenau. Quellenschriften der Elsässischen Kirchengeschichte 5. Straßburg: Le Roux, I 898.

Helvetia Sacra. Abteilung 4, Bd. 7, Teil I. Die Johanniter, die Templer, der Deutsche Orden. die Lazariter und Lazariterinnen, die Pauliner und die Serviten in der Schweiz. Herausgegeben von Petra Zimmer und Patrick Braun. Basel: Schwabe, 2006.

Hessisches Klosterbuch: Quellenkunde zur Geschichte der im Regierungsbezirk Kassel, im Kreis Grafschaft Schaumburg, der Provinz Oberhessen und dem Kreis Biedenkopf gegründeten Stifter, Klöster und Niederlassungen von geistlichen Genossenschaften. Herausgegeben von Wilhelm Dersch. Veröffentlichungen der Historischen Kommission für Hessen und Waldeck I 2. Marburg: Elwert, I 940, 2. Auflage.

Regesta episcoporum Constantiensium. Bd. 3. 1384-1436. Herausgegeben von Karl Rieder. Innsbruck: Wagner, 1926.

Sources Concerning the Hospitallers of St John in the Netherlands 14th-18th Centuries. Herausgegeben von Johanna Maria van Winter. Studies in the History of Christian Thought 89. Leiden-Boston-Köln: Brill, 1998.

Die Würzburger Inschriften bis I 525. Herausgegeben von Karl Borchardt und Franz Xaver Herrmann. Die Deutschen Inschriften 27. Wiesbaden: Reichert, 1988.

\section{SECONDARY SOURCES:}

Ahn, Sang-Joon. Die Kölner Johanniterkommende Sankt Johann und Cordula im Spätmittelalter: Geschichte, Besitz, Wirtschaft und Sozialstruktur. Kölner Schriften zu Geschichte und Kultur 28. Köln: SH-Verlag, 2006.

Armgart, Martin. "Die Johanniter von Heimbach: Regionale und überregionale Verbindungen der südpfälzischen Kommende eines Ritterordens während des Mittelalters." Zeitschrift für Geschichte des Oberrheins 164 (2016): I I I-I 52.

Bendel, Franz Josef. "Die Würzburger Diözesanmatrikel aus der Mitte des I 5. Jahrhunderts." Würzburger Diözesangeschichtsblätter 2/2 (1934): I-XXX, I-46. 
Borchardt, Karl. "Die Johanniter in Deutschland und die Reformation." In The Military Orders and the Reformation: Choices, State Building, and the Weight of Tradition, Papers of the Utrecht Conference, 30 September - 2 October 2004, herausgegeben von Johannes A. Mol, Klaus Militzer, und Helen J. Nicholson, I I- I I 7. Bijdragen tot de Geschiedenis van de Ridderlijke Duitsche Orde, Balije van Utrecht 3. Hilversum: Verloren, 2006.

Borchardt, Karl. "Hospitaller Chapters in the Medieval Priory of Alamania." In Piety, Pugnacity and Property, herausgegeben von Nicholas Morton, 195-207. The Military Orders 7. London: Routledge, 2019.

Borchardt, Karl. "Reichardsroth zur Zeit der Johanniter." In Kirchen, Bürger und Bauern: Ausgewählte Studien zu Rothenburg ob der Tauber und seinem Umland, herausgegeben von Karl Borchardt, I 24-I39. Jahrbuch des Vereins Alt-Rothenburg. Rothenburg: Verein Alt-Rothenburg, 20 I6. Zuerst erschienen: Reichardsroth und sein Johannitererbe 1254-2004. Herausgegeben von Ev.-Luth. Pfarramt Langensteinach, 54-65. Uffenheim: Ev.-Luth. Pfarramt Langensteinach, 2004.

Borchardt, Karl. "Soll-Zahlen zum Personalstand der deutschen Johanniter vom Jahre I 367." Revue Mabillon 75, Nr. I 4 (2003): 83- I 13.

Borchardt, Karl. “Tätigkeit und Karrieren von Johannitern in der Ballei Wetterau." In Johanniter in Hessen. 800 Jabre diakonischer Auftrag, herausgegeben von Reiner Braun und Ruth Slenczka, 17-33. Jahrbuch der Hessischen Kirchengeschichtlichen Vereinigung 59. Darmstadt: Hess. Kirchengeschichtl. Vereinigung, 2008.

Borchardt, Karl. Die geistlichen Institutionen in der Reichsstadt Rothenburg ob der Tauber und dem zugehörigen Landgebiet von den Anfängen bis zur Reformation. Veröffentlichungen der Gesellschaft für fränkische Geschichte IX/37. 2 Tle. Neustadt/Aisch: Degener, 1988.

Bünz, Enno. Die mittelalterliche Pfarrei. Ausgewählte Studien zum I3.-I6. Jabrhundert. Spätmittelalter, Humanismus, Reformation 96. Tübingen: Mohr Siebeck, 2017.

Die Pfarrei im späten Mittelalter. Herausgegeben von Enno Bünz und Gerhard Fouquet. Vorträge und Forschungen 77. Ostfildern: Thorbecke, 20 I 3.

Gahlbeck, Christian. "Strukturreform um der Wirtschaftlichkeit willen? Die Ballei Brandenburg der Johanniter unter Hermann von Warberg nach I 350 ." Jahrbuch für die Geschichte Mittel-und Ostdeutschlands 62 (2016): 97-I 34.

Huschner, Wolfgang, Ernst Münch und Cornelia Neustadt. Mecklenburgisches Klosterbuch: Handbuch der Klöster, Stifte, Kommenden und Prioreien (Io./II.-I6. Jahrhundert). 2 Bde. Rostock: Hinstorff, 2016.

Krämer, Thomas. Dämonen, Prälaten und gottlose Menschen. Konflikte und ibre Beilegung im Umfeld der geistlichen Ritterorden. Vita regularis 64. Berlin-Münster: LIT, 20 I 5.

Krebs, Manfred. "Die Annaten-Register des Bistums Konstanz aus dem I 5 . Jahrhundert." Freiburger Diözesanarchiv 76 (1956): I-467.

Krebs, Manfred. "Die Annatenregister des Bistums Konstanz und ihre Bedeutung für die württembergische Kirchengeschichte." Zeitschrift für württembergische Landesgeschichte I 3 (1954): I09-I I9. 
Krebs, Manfred. "Die Investiturprotokolle der Diözese Konstanz aus dem I 5. Jahrhundert." Freiburger Diözesan-Archiv 66 (1938): Anhang I-104; 67 (1940): Anhang 105-264; 68 (194I): Anhang 265-424; 70 (1950): Anhang 425-546; 7 I (1951): Anhang 547-642; 72 (1952): Anhang 643-786; 73 (1953): Anhang 787-1 047; 74 (1954): Anhang I-160 [Register].

Landau, Peter. Ius patronatus. Studien zur Entwicklung des Patronats im Dekretalenrecht und in der Kanonistik des I2. und I3. Jahrbunderts. Forschungen zur kirchlichen Rechtsgeschichte und zum Kirchenrecht I 2. Köln-Wien: Böhlau, I 975.

Lindenhayn-Fiedorowicz, Agnieszka. "Johannitisches Patronat und städtische Architektur: Die Marienkirche zu Stargard (Stargard Szczeciński) in Pommerns." In Regionalität und Transfergeschichte: Ritterordenskommenden der Templer und Johanniter im nordöstlichen Deutschland und in Polen, herausgegeben von Christian Gahlbeck, Heinz-Dieter Heimann, und Dirk Schumann, 248-270. Studien zur brandenburgischen und vergleichenden Landesgeschichte 9, Schriften der Landesgeschichtlichen Vereinigung für die Mark Brandenburg N.F. 4 Berlin: Lukas, 20 I 4.

Luckhardt, Jochen. "Zur Bildausstattung des Totenbuches der Johanniterkommende Burgsteinfurt." In Masters and Miniatures. Proceedings of the Congress on Medieval Manuscript Illumination in the Northern Netherlands (Utrecht, Io-I3 December 1989), herausgegeben von Koert van der Horst und Johann-Christian Klamt, I95-200. Studies and Facsimiles of Netherlandish Illuminated Manuscripts 3. Doornspijk: Davaco, I 99 I.

Niedersächsisches Klosterbuch: Verzeichnis der Klöster, Stifte, Kommenden und Beginenhäuser in Niedersachsen und Bremen von den Anfängen bis I8Io. 4 Bde. Herausgegeben von Josef Dolle. Bielefeld: Verlag für Regionalgeschichte, 20 I 2.

Nordrheinisches Klosterbuch: Lexikon der Stifte und Klöster bis I8I5. Teil I. Aachen bis Düren. Herausgegeben von Manfred Groten. Siegburg: Franz Schmitt, 2009.

Nordrheinisches Klosterbuch: Lexikon der Stifte und Klöster bis I8I5. Teil 2. Düsseldorf bis Kleve. Herausgegeben von Manfred Groten. Siegburg: Franz Schmitt, 20 I 2.

Osterhausen, Christian von. Eigentlicher und gründlicher Bericht dessen was zu einer volkommenen Erkantnuß deß hochlöblichen Ritterlichen Ordens $S$. Johannis von Jerusalem zu Malta vonnöthen. Augsburg: Andreas Aperger, 1650.

Petke, Wolfgang. "Von der klösterlichen Eigenkirche zur Inkorporation in Lothringen und Nordfrankreich im I I. und I 2. Jahrhundert." Revue d'histoire ecclésiastique 87 ( 1992 ): 34-72, 375-404.

Rödel, Walter G. Das Großpriorat Deutschland des Johanniter-Ordens im Übergang vom Mittelalter zur Reformation anhand der Generalvisitationsberichte von 1494/95 und I540/4I. Köln: Wienand, I 972, 2 Auflage.

Sägmüller, Johannes Baptist. Lehrbuch des katholischen Kirchenrechts. 2 Bde. Freiburg im Breisgau: Herder, I 9 I 4, 3. Auflage.

Sgonina, Marc. Die Johanniterballei Westfalen: Unter besonderer Berücksichtigung der Lebensformen der Zentralkommende Steinfurt und ibrer Membra. Frankfurt/Main: Lang, 20 I 4. 
Smoliński, Marek. "Zur Geschichte der Johanniter und ihrer politischen Rolle in Polen bis zum Jahre I 370." In Regionalität und Transfergeschichte: Ritterordenskommenden der Templer und Johanniter im nordöstlichen Deutschland und in Polen, herausgegeben von Christian Gahlbeck, Heinz-Dieter Heimann, und Dirk Schumann, 77-91. Studien zur brandenburgischen und vergleichenden Landesgeschichte 9, Schriften der Landesgeschichtlichen Vereinigung für die Mark Brandenburg N.F. 4 Berlin: Lukas, 2014.

Stälin, Paul Friedrich. "Komture des Johanniter-Ordens im Gebiet des jetzigen Königreichs Württemberg." Archivalische Zeitschrift I/8 ( I 883): I 06 - I I I.

Szczesiak, Rainer, und Christian Gahlbeck, "Die Kommenden der Johanniter in Mirow, Gardow und Nemerow in der Herrschaft Stargard in Südmecklenburg." In Regionalität und Transfergeschichte: Ritterordenskommenden der Templer und Johanniter im nordöstlichen Deutschland und in Polen, herausgegeben von Christian Gahlbeck, Heinz-Dieter Heimann, und Dirk Schumann, 204-247. Studien zur brandenburgischen und vergleichenden Landesgeschichte 9, Schriften der Landesgeschichtlichen Vereinigung für die Mark Brandenburg N.F. 4. Berlin: Lukas, 20 I 4.

Westfälisches Klosterbuch: Lexikon der vor I 8 I errichteten Stifte und Klöster von ihrer Gründung bis zur Aufhebung. Herausgegeben von Karl Hengst. Veröffentlichungen der Historischen Kommission für Westfalen 44/ I -3. Münster: Aschendorff, I 992/2003.

Württembergisches Klosterbuch: Klöster, Stifte und Ordensgemeinschaften von den Anfängen bis in die Gegenwart. Herausgegeben von Wolfgang Zimmermann und Nicole Piersching. Ostfildern: Thorbecke, 2003.

Zwehl, Hans Karl von. Über die Entwicklung der Adelsproben im Souveränen Malteser-Ritterorden: Eine Studie. Essen: Fredebeul \& Koenen, 1932. 